\title{
Stimulation of Entorhinal Cortex Promotes Adult Neurogenesis and Facilitates Spatial Memory
}

\author{
Scellig S. D. Stone, ${ }^{1,2,3,5}$ Cátia M. Teixeira, ${ }^{1,6}$ Loren M. DeVito, ${ }^{1}$ Kirill Zaslavsky, ${ }^{1}$ Sheena A. Josselyn, ${ }^{1,2,4}$ \\ Andres M. Lozano, ${ }^{2,3,5}$ and Paul W. Frankland ${ }^{1,2,4}$ \\ ${ }^{1}$ Program in Neurosciences and Mental Health, The Hospital for Sick Children, Toronto, Ontario, Canada M5G 1X8, ${ }^{2}$ Institute of Medical Science, ${ }^{3}$ Division \\ of Neurosurgery, and ${ }^{4}$ Department of Physiology, University of Toronto, Toronto, Ontario, Canada, M5S 1A8, ${ }^{5}$ Division of Neurosurgery, Toronto Western \\ Hospital, Toronto, Ontario, Canada, M5T 2S8, and ${ }^{6}$ Graduate Program in Areas of Basic and Applied Biology, University of Porto, 4050-465 Porto, Portugal
}

Deep brain stimulation (DBS) is an established therapeutic modality for the treatment of movement disorders and an emerging therapeutic approach for the treatment of disorders of mood and thought. For example, recently we have shown that DBS of the fornix may ameliorate cognitive decline associated with dementia. However, like other applications of DBS, the mechanisms mediating these clinical effects are unknown. As DBS modulates neurophysiological activity in targeted brain regions, DBS might influence cognitive function via activity-dependent regulation of hippocampal neurogenesis. Using stimulation parameters analogous to clinical high-frequency DBS, here we addressed this question in mice. We found that acute stimulation of the entorhinal cortex (EC) transiently promoted proliferation in the dentate gyrus (DG). Cells generated as a consequence of stimulation differentiated into neurons, survived for at least several weeks, and acquired normal dentate granule cell (DGC) morphology. Importantly, stimulation-induced promotion of neurogenesis was limited to the DG and not associated with changes in apoptotic cell death. Using immunohistochemical approaches, we found that, once sufficiently mature, these stimulation-induced neurons integrated into hippocampal circuits supporting water-maze memory. Finally, formation of water-maze memory was facilitated 6 weeks (but not 1 week) after bilateral stimulation of the EC. The delay-dependent nature of these effects matches the maturation-dependent integration of adult-generated DGCs into dentate circuits supporting water-maze memory. Furthermore, because the beneficial effects of EC stimulation were prevented by blocking neurogenesis, this suggests a causal relationship between stimulation-induced promotion of adult neurogenesis and enhanced spatial memory.

\section{Introduction}

Deep brain stimulation (DBS) uses surgically implanted electrodes to deliver electrical stimulation to precisely targeted areas in the brain. To date, $>60,000$ patients have been implanted with deep brain electrodes (Ponce and Lozano, 2010), and its predominant application has been in the treatment of movement disorders, most commonly Parkinson's disease (Koller et al., 1997; Vidailhet et al., 2005; Deuschl et al., 2006). However, studies have also begun to explore its potential application to a widening array of neurologic and psychiatric conditions, including disorders of

Received June 18, 2011; revised July 25, 2011; accepted July 29, 2011.

Author contributions: S.S.D.S., S.A.J., A.M.L., and P.W.F. designed research; S.S.D.S., C.M.T., L.M.D., and K.Z. performed research; S.S.D.S., L.M.D., and S.A.J. analyzed data; S.S.D.S., A.M.L., and P.W.F. wrote the paper.

The authors declare no conflicts of interest.

This work was supported by Canadian Institutes of Health Research Grants MOP-86762 and IAP-102234 (P.W.F.). S.S.D.S. was supported by a Canadian Institutes of Health Research Fellowship, the Department of Surgery's Surgeon Scientist Program at the University of Toronto, and a Michael J. Fox Foundation Fellowship. C.M.T. received support from the Graduate Program in Areas of Basic and Applied Biology and the Portuguese Foundation for Science and Technology. S.A.J. holds a Tier 2 Canada Research Chair in Molecular and Cellular Cognition. A.M.L. holds a Tier 1 Canada Research Chair in Neuroscience and the R.R. Tasker Chair in Functional Neurosurgery at the University of Toronto. P.W.F. holds a Tier 2 Canada Research Chair in Cognitive Neurobiology. We thank Berge Minassian for providing tracer compound and Masanori Sakaguchi and Maithe Arruda-Carvalho for technical assistance.

Correspondence should be addressed to Paul W. Frankland, Neurosciences and Mental Health, The Hospital for Sick Children, 555 University Avenue, 6018 McMaster Building, Toronto, ON, Canada, M5G 1X8. E-mail: paul.frankland@sickkids.ca.

DOI:10.1523/JNEUROSCI.3100-11.2011

Copyright $\odot 2011$ the authors $\quad 0270-6474 / 11 / 3113469-16 \$ 15.00 / 0$ mood and thought. For example, DBS of limbic circuits has shown promise in treating refractory depression (Mayberg et al., 2005), whereas DBS of basal ganglia circuitry has been used in the treatment of obsessive compulsive disorder (Mallet et al., 2008) and Tourette syndrome (Shahed et al., 2007). Moreover, we recently reported a phase 1 trial investigating DBS of the limbic system for the treatment of dementia/cognitive impairment (Laxton et al., 2010). In this trial, DBS of the fornix was associated with arrest or slowing of cognitive decline in certain patients, measured by two different scales of cognitive functioning. Although the mechanism(s) of action underlying these clinical effects, and DBS in general, remain(s) poorly understood (Kringelbach et al., 2007), it is clear that DBS modulates the activity of targeted brain circuits (Davis et al., 1997; Mayberg et al., 2005). For instance, in our trial, fornix stimulation strongly activated the hippocampus and parahippocampal gyrus, including the entorhinal cortex (EC) (Laxton et al., 2010).

One potential mechanism through which DBS might influence cognitive function is via activity-dependent regulation of hippocampal neurogenesis. New neurons are continuously added to the adult mammalian dentate gyrus (DG), a subregion of the hippocampus that plays an essential role in memory formation (Ming and Song, 2005; Zhao et al., 2008). These adult-generated dentate granule cells (DGCs) eventually develop similar cellular phenotypes to developmentally generated DGCs (Laplagne et al., 2006, 2007), and, once sufficiently mature, are thought to contribute to the for- 
mation of hippocampus-dependent memory (Shors, 2008; Deng et al., 2010). Consistent with the idea that activity regulates neuron production in the adult DG, stimulation of limbic targets in rodents [e.g., the anterior thalamic nucleus (Toda et al., 2008; Encinas et al., 2011), EC/perforant path (Bruel-Jungerman et al., 2006; Chun et al., 2006; Kitamura et al., 2010), or mossy fibers (Derrick et al., 2000)] promotes the proliferation and/or survival of adult-generated DGCs in vivo. Although these studies demonstrate that stimulation of hippocampal afferents can reliably increase the production of new neurons, whether these stimulation-induced increases in neurogenesis impact hippocampal function is not known. Our experiments indicate that new granule cells, born as a consequence of EC stimulation, mature normally and assume functional roles in hippocampal circuits supporting spatial memory. Furthermore, stimulationinduced promotion of hippocampal neurogenesis facilitates spatial memory formation, suggesting that enhanced neurogenesis is one mechanism via which DBS may have pro-cognitive effects.

\section{Materials and Methods}

Mice. Male offspring from a cross between C57BL/6NTacfBr and 129 Svev mice (Taconic) were used in these experiments. All mice were bred in the colony at The Hospital for Sick Children and housed in groups of three to five mice per cage. They were maintained on a $12 \mathrm{~h}$ light/dark cycle with access to food and water ad libitum and were 8 weeks old at the start of experiments. Behavioral procedures were conducted during the light phase of the cycle, blind to the treatment condition of the mouse and according to protocols approved by the Animal Care Committee at The Hospital for Sick Children.

Stereotactic surgery. Mice were pretreated with atropine sulfate $(0.1$ $\mathrm{mg} / \mathrm{kg}$, i.p.), anesthetized with chloral hydrate (400 mg/kg, i.p.), and placed in a stereotactic frame. The scalp was incised and skull hole(s) drilled. Targets with coordinates relative to bregma in the anteroposterior, mediolateral, and dorsoventral planes were as follows (in $\mathrm{mm}$ ): (1) EC bordering on the lateral and medial entorhinal areas, specifically near the junction of the ventral intermediate, medial, and caudal entorhinal fields (van Groen, 2001) [-4.0, 3.0, 5.1]; (2) DG [-2.0, 1.6, 2.0]; (3) fourth and fifth cerebellar lobules $[-6.2,1.0,2.0]$ (Paxinos and Franklin, 2000).

Fluorescent anterograde tracer labeling. An anterograde tracer (fluorescent-labeled $10 \mathrm{kDa}$ dextran [fluoro-ruby D-1817; Invitrogen]; 0.82 $\mathrm{mg} / \mathrm{ml}$ ) was injected unilaterally into the EC via a glass micropipette (outer diameter, $\sim 50 \mu \mathrm{m}$ ) connected to a Hamilton syringe. Injection volume $(1.5 \mu \mathrm{l})$ and rate $(0.15 \mu \mathrm{l} / \mathrm{min})$ were controlled by an infusion pump (SP100i; WPI). The micropipette was left in place for an additional 10 min to ensure complete diffusion of tracer.

Brain electrical stimulation. Electrical stimulation was delivered via concentric bipolar electrodes (CBASC75; FHC), the detailed electrical properties of which have been described previously (Gimsa et al., 2005, 2006). Electrode integrity was verified by impedance measurement. Stacked contacts include a $100-\mu \mathrm{m}$-long $\times 125-\mu \mathrm{m}$-diameter stainless steel outer pole, $100 \mu \mathrm{m}$ separation, and $100-\mu \mathrm{m}$-long $\times 25-\mu \mathrm{m}$ diameter platinum/iridium inner pole. Targeting accuracy was refined to $\pm 0.5 \mathrm{~mm}$ in the anteroposterior and mediolateral planes and $\pm 0.25 \mathrm{~mm}$ in the dorsoventral plane during pilot experiments. Stimulation was applied with a clinical screener (model 3628; Medtronic) using frequency $(130 \mathrm{~Hz})$ and pulse width $(90 \mu \mathrm{s}$, square wave) settings approximating high-frequency DBS used in clinical practice (Volkmann et al., 2006). Current $(0-500 \mu \mathrm{A})$, duration $(30-120 \mathrm{~min})$, and laterality (unilateral or bilateral) of stimulation varied by experiment. Charge density, calculated using electrode geometry (McCreery et al., 1990; Gimsa et al., 2005, 2006), was kept below $30 \mu \mathrm{C} / \mathrm{cm}^{2} /$ phase to minimize the risk of stimulation-induced neural injury (McCreery et al., 1990).

Retroviral labeling of new neurons. New neurons were labeled by CAG promoter-driven green fluorescent protein (GFP) expression after infection with a replication-deficient retroviral vector (based on the Moloney murine leukemia virus) (Tashiro et al., 2006). Viral vector was prepared by transfecting Platinum-gp cells with two plasmids containing an amphotropic envelope (vesicular stomatitis virus-glycoprotein) and the transgene (pCAG-GFP), followed by collection through ultraspeed centrifugation. Platinum-E cells were then infected to generate a stable virusproducing cell line, and concentrated virus solution was obtained by ultraspeed centrifugation (average of $3.5 \times 10^{9} \mathrm{IU} / \mathrm{ml}$ ). Viral vectors were injected bilaterally into the DG via a glass micropipette (outer diameter, $\sim 50 \mu \mathrm{m}$ ) connected to a Hamilton syringe. Injection volume $(1.5 \mu \mathrm{l} / \mathrm{side})$ and rate $(0.12 \mu \mathrm{l} / \mathrm{min})$ were controlled by an infusion pump (SP100i; WPI). Micropipettes were left in place for an additional $10 \mathrm{~min}$ to ensure complete diffusion of virus.

$B r d U, C l d U$, and IdU administration. The thymidine analogs 5-bromo-2' deoxyuridine (BrdU; Sigma), 5-iodo-2' -deoxyuridine (IdU; MP Biomedicals), or 5-chloro-2'-deoxyuridine (CldU; Sigma) were dissolved in $0.1 \mathrm{~m}$ $\mathrm{PBS}$ and heated to $50-60^{\circ} \mathrm{C}$, at a concentration of $10 \mathrm{mg} / \mathrm{ml}$. Intraperitoneal $\mathrm{BrdU}$ injection dosages and dosing intervals are described below (see Experimental procedures). IdU and CldU were given intraperitoneally at equimolar concentrations to $50 \mathrm{mg} / \mathrm{kg} \mathrm{BrdU}$ (corresponding to $57.5 \mathrm{mg} / \mathrm{kg}$ IdU and $42.5 \mathrm{mg} / \mathrm{kg} \mathrm{CldU}$ ) three times per day ( $8 \mathrm{~h}$ apart) for 3 consecutive days as indicated below (see Experimental procedures).

Temozolomide administration. Temozolomide (TMZ) (Sigma) was dissolved in dimethylsulfoxide (DMSO), diluted in PBS to a concentration of $2.5 \mathrm{mg} / \mathrm{ml}$ (10\% DMSO), and administered intraperitoneally at a dose of $25 \mathrm{mg} / \mathrm{kg}$ once daily for 3 consecutive days according to a previous protocol (Garthe et al., 2009). Vehicle solution (V) was the identical $\mathrm{DMSO} / \mathrm{PBS}$ solution but without TMZ and was administered in volumes consistent with TMZ dosing.

Water-maze apparatus and procedures. The apparatus and behavioral procedures have been described previously (Teixeira et al., 2006). In brief, a circular water-maze tank ( $120 \mathrm{~cm}$ diameter, $50 \mathrm{~cm}$ deep), located in a dimly lit room, was filled to a depth of $40 \mathrm{~cm}$ with water (maintained at $28 \pm 1{ }^{\circ} \mathrm{C}$ and made opaque by adding white, nontoxic paint). A circular escape platform $(10 \mathrm{~cm}$ diameter $)$ was submerged $0.5 \mathrm{~cm}$ below the water surface, in a fixed position in one quadrant. White curtains with distinct cues painted on them surrounded the pool, each $\geq 1 \mathrm{~m}$ from the pool perimeter.

Before training, mice were individually handled for 2 min each day over 7 consecutive days. Mice were trained with one of two protocols: standard training over $5 \mathrm{~d}$ with six training trials per $\mathrm{d}$ (presented in two blocks of three trials; interblock interval, $\sim 1 \mathrm{~h}$; intertrial interval, $\sim 15 \mathrm{~s}$ ) or undertraining over $3 \mathrm{~d}$ with three trials per day (intertrial interval, $\sim 15 \mathrm{~s}$ ). On each trial, mice were placed into the pool, facing the wall, in one of four start locations (the order of which was pseudorandomly varied throughout training). The trial was complete once the mouse found the platform or $60 \mathrm{~s}$ had elapsed. If the mouse failed to find the platform on a given trial, the experimenter guided the mouse onto the platform. After the completion of training, spatial memory was assessed in a series of three probe tests with an intertest interval of $\sim 3 \mathrm{~min}$. In this test, the platform was removed from the pool, and the mouse was allowed $60 \mathrm{~s}$ to search for it.

Behavioral data from training trials and probe tests were acquired and analyzed using an automated tracking system (Actimetrics). General training measures included latency to reach the platform and path length. Search strategies during training were analyzed using numerical parameters from swim tracking data (adapted from Garthe et al., 2009), and the respective predominant search strategy for each trial was objectively classified by a criterion-based algorithm. Trials were classified as one of the following ordered strategies (tracking criteria in parentheses): (1) "direct swim," characterized by a maintained heading toward the platform $\left(<15 \mathrm{~cm}\right.$ path length or heading $<22.5^{\circ}$ away from platform at each $5 \mathrm{~cm}$ point starting at $15 \mathrm{~cm}$ path length); (2) "focal search," characterized by highly localized search near the platform $(>50 \%$ trial in 15 $\mathrm{cm}$ radius target zone, centered on the platform location); (3) "directed search," characterized by a preference for a corridor toward the platform or platform quadrant $(<50 \%$ trial outside a 50 -cm-wide corridor from start point to platform and $>20 \%$ in $25 \mathrm{~cm}$ target zone, or $>40 \%$ in target quadrant); (4) "chaining," characterized by searching near the correct radial distance of the platform to the wall $(>75 \%$ trial $20-50 \mathrm{~cm}$ from pool center, $<15 \%$ within $10 \mathrm{~cm}$ of wall, and $<10 \%$ within $20 \mathrm{~cm}$ of pool center); (5) "scanning," characterized by a preference for the central pool area in which distal cue visibility is maximal ( $>50 \%$ trial within 35 
cm of pool center); (6) "thigmotaxis," characterized by maintaining close proximity to the wall ( $>70 \%$ trial within $10 \mathrm{~cm}$ of wall); (7) "perseverance," characterized by an erroneous preference for a non-target area ( $>60 \%$ trial in one or $>75 \%$ in two adjacent non-target quadrant(s) and $>750 \mathrm{~cm}$ path length); and (8) "random search," characterized by no other discernable strategy (remaining unclassified trials).

Probe test performance was quantified in three ways: (1) the amount of time mice searched the target zone versus the average of the three other equivalent zones in other areas of the pool [zone radius was either 15 or $20 \mathrm{~cm}$ (for the TMZ experiment)], (2) the number of platform location crossings versus the average of the three other equivalent platform locations in other areas of the pool, and (3) the frequency with which mice visited areas of the pool represented as a density plot (or heat map, with hot colors corresponding to more frequent visits) generated using Matlab (MathWorks).

Immunohistochemistry. Mice were perfused transcardially with $0.1 \mathrm{M}$ PBS and $4 \%$ paraformaldehyde (PFA). Brains were removed, fixed overnight in PFA, and transferred to $0.1 \mathrm{M}$ PBS. Fifty micrometer horizontal (for some tracer analyses) or coronal sections were cut using a vibratome (VT1200S; Leica). TUNEL staining was performed using the DeadEnd Calorimetric TUNEL System (Promega) and cresyl violet counterstaining. For other analyses, the following primary antibodies were used: rabbit polyclonal anti-Fos (1:1000; Calbiochem), rat monoclonal anti-BrdU for BrdU and CldU specifically (1:500; Accurate Chemicals), mouse monoclonal anti-BrdU for IdU specifically (1:1000; BD Biosciences), mouse monoclonal anti-NeuN (1:1000; Millipore Bioscience Research Reagents), rabbit polyclonal anti-GFP (1:500; Invitrogen), and Alexa Fluor 488-conjugated mouse monoclonal anti-NeuN (1:1000; Millipore Bioscience Research Reagents). Detailed BrdU/IdU/CldU/Fos/ NeuN staining procedures have been described previously (Stone et al., 2010). In brief, sections were washed and incubated for $48 \mathrm{~h}$ at $4^{\circ} \mathrm{C}$ with primary antibodies and then for $2 \mathrm{~h}$ at $20^{\circ} \mathrm{C}$ with the following fluorescent-conjugated or biotinylated secondary antibodies: Alexa Fluor-488 anti-rat (1:500; Invitrogen), Alexa Fluor-568 anti-mouse (1:500; Invitrogen), Alexa Fluor-568 anti-rabbit (1:500; Invitrogen), Rhodamine Red-X-conjugated anti-mouse (1:500; Jackson ImmunoResearch), FITC-conjugated anti-rat (1:500; Jackson ImmunoResearch), or Biotin-SP-conjugated anti-rabbit (1:500; Jackson ImmunoResearch). Biotinylated antibody signals were visualized using Alexa Fluor-conjugated streptavidin (Invitrogen). Sections were slide mounted with Permafluor anti-fade medium (Lipshaw Immunon).

Imaging and quantification. All images were acquired using epifluorescent (BX61; Olympus) or confocal laser-scanning (LSM710; Carl Zeiss) microscopes and displayed as maximum-intensity projections of $Z$-stack images created using Image-Pro (Media Cybernetics) or ZEN (Carl Zeiss) software. A laser pinhole setting of 1 Airy unit and $Z$-stack image spacing of $0.5-1.0 \mu \mathrm{m}$ (spanning the regions of interest) were used for all confocal imaging. These produced serial optical slices of $0.8 \mu \mathrm{m}$ and 1.0 $\mu \mathrm{m}$ under $100 \times$ and $40 \times$ objectives, respectively.

Fluorescent tracer distribution was qualitatively assessed from survey images acquired using $10-40 \times$ objectives on the epifluorescent microscope. The presence of tracer within neuronal projections was verified using confocal microscopy. DG regional analysis was performed using serial sections covering the entire anteroposterior extent of the DG, grouped by thirds as anterior, middle, or posterior.

$\mathrm{BrdU}^{+}, \mathrm{CldU}^{+}, \mathrm{IdU}^{+}, \mathrm{GFP}^{+}, \mathrm{Fos}^{+}$, and $\mathrm{CldU}^{+}$or $\mathrm{IdU}^{+}\left(\mathrm{XdU}^{+}\right) /$ $\mathrm{Fos}^{+}$cells were quantified using a $40 \times$ objective on the epifluorescent microscope. DG total cell numbers were estimated stereologically per side using systematic random sampling fractions of $1 / 4$ serially cut coronal sections covering the entire anteroposterior extent of the DG. The tops of nuclei served as unique characteristic points for exhaustive direct cell counting using Stereo Investigator (MicroBrightField) software. Sample totals were then multiplied by the inverse sampling rate (4) to give total DG estimates. After identification under the epifluorescent microscope, apparent double-labeled cells were confirmed by confocal microscopy.

Area densities of subventricular zone (SVZ) BrdU ${ }^{+}$cells were derived using a method modified from Bath et al. (2008). A systematic random sample fraction of $1 / 4$ serially cut coronal sections, covering $\sim 1.54-0.50$ $\mathrm{mm}$ relative to bregma, was used. All $\mathrm{BrdU}^{+}$nuclei within $0.1 \mathrm{~mm}$ of the lateral ventricle ependymal surfaces were counted per side, marking the tops of nuclei as unique characteristic counting points, using a $40 \times$ objective on the epifluorescent microscope and Stereo Investigator software. For each section counted, reference lines tracing each ependymal surface were drawn using Stereo Investigator software. Area densities were then represented by dividing total cell counts by total reference line lengths for each side [giving $\mathrm{BrdU}^{+}$cells $/(1.0 \mathrm{~mm}$ reference line $\times 0.1$ $\mathrm{mm}$ subventricular zone depth), or BrdU ${ }^{+}$cells $/ 0.1 \mathrm{~mm}^{2}$ ].

To estimate the proportions of $\mathrm{IdU}^{+}$and $\mathrm{CldU}^{+}$cells that were colabeled with NeuN after unilateral surgery, DG regions of interest were randomly selected from five mice and analyzed using confocal microscopy. A total of $300 \mathrm{IdU}^{+}$and $300 \mathrm{CldU}^{+}$cells were analyzed (i.e., 30 cells per side per mouse). The proportions of $\mathrm{BrdU}^{+}$cells colabeled with NeuN after bilateral surgery and drug treatment were identically derived from five to eight mice per group.

Neuron tracings and spine counts were manually collected from 0.5 $\mu \mathrm{m}$ spaced $Z$-stack images acquired with a $100 \times$ objective on the epifluorescent microscope and using Neurolucida (MicroBrightField) software. In each of three mice in the 6 week group, six ipsilateral and six contralateral neurons were analyzed (for a total of 18 ipsilateral and 18 contralateral neurons from three mice). Dendritic architecture and spines were quantified for each neuron in its entirety (i.e., spines were counted along the entire dendrite length and on all dendrite branches for each neuron). Dendritic architecture was classified as aberrant if there was a dendritic process with spines pointing toward the hilus, and granule cells were considered ectopic if located more than two cell layers away from the inner border of the granule cell layer (Jessberger et al., 2007). Total dendrite length, nodes, and linear spine density (total spines divided by total dendrite length for each branch order) were quantified using Neurolucida software. Axon distribution was qualitatively assessed from survey images of the CA3 region using confocal microscopy.

Experimental procedures. To characterize EC projections to the DG, mice received unilateral injections of fluorescent tracer into the EC ( $n=$ 5) and were perfused $7 \mathrm{~d}$ later (Table 1).

Stimulation-induced DG activation was assessed by placing mice $(n=$ 6) under anesthesia for $3 \mathrm{~h}$ before surgery. Unilateral EC stimulation was delivered for $1 \mathrm{~h}(50 \mu \mathrm{A})$. Anesthesia was maintained for an additional hour before perfusion.

Stimulation-induced effects on DG proliferation and apoptosis were determined after unilateral EC stimulation ( $50 \mu \mathrm{A}$ for $1 \mathrm{~h}$ ). After stimulation, mice were treated with a single $200 \mathrm{mg} / \mathrm{kg} \mathrm{BrdU}$ injection $1(n=$ $5), 3(n=4), 5(n=4)$, or $7(n=4) \mathrm{d}$ later and perfused $24 \mathrm{~h}$ after injection. Additional nonstimulated mice underwent electrode insertion, with no current delivery, followed by BrdU $1(n=5), 3(n=6), 5(n=4)$, or $7(n=4)$ d postoperatively.

To examine the effects of varied stimulation durations and currents on cell proliferation, mice received unilateral EC stimulation. Three days later, they received a single $200 \mathrm{mg} / \mathrm{kg}$ BrdU injection and were perfused $24 \mathrm{~h}$ later. To examine the impact of stimulation duration on proliferation, $50 \mu \mathrm{A}$ was delivered for $30(n=5), 60(n=4)$, or $120(n=5) \mathrm{min}$. To examine the effect of current intensity on proliferation, mice received $50(n=4), 250(n=5)$, or $500(n=5) \mu$ A stimulation for $1 \mathrm{~h}$.

The proportion of stimulation-induced newly born cells adopting a neuronal phenotype was assessed after unilateral EC stimulation $(50 \mu \mathrm{A}$ for $1 \mathrm{~h}, n=5$ ). Mice received IdU injections during the period of stimulation-induced increased proliferation (postoperative days $3-5$ ), CldU injections during a similar period of baseline proliferation (postoperative days 7-9), and were perfused $\sim 10$ weeks later.

To assess stimulation-induced effects on cell survival, mice were treated twice daily with $100 \mathrm{mg} / \mathrm{kg}$ BrdU injections ( $8 \mathrm{~h}$ apart) for 3 consecutive days, 1,10 , or $30 \mathrm{~d}$ ( $n=8$ each) before unilateral EC stimulation ( $50 \mu \mathrm{A}$ for $1 \mathrm{~h}$ ), and perfused 3 weeks postoperatively.

Subventricular zone proliferation after unilateral EC stimulation and DG proliferation after unilateral cerebellar stimulation were assessed in separate experiments using single $200 \mathrm{mg} / \mathrm{kg}$ BrdU injections $3 \mathrm{~d}$ after stimulation (50 $\mu \mathrm{A}$ for $1 \mathrm{~h}$ ). Both experiments included stimulated and nonstimulated groups ( $n=4$ each) perfused $24 \mathrm{~h}$ after BrdU injection.

Retroviral-mediated labeling of adult-born DGCs was performed by bilateral viral solution injections into the DG $3 \mathrm{~d}$ after unilateral EC 
Table 1. Experimental procedures

\begin{tabular}{|c|c|c|c|c|c|}
\hline Experiment (Figure) & Treatment & Cell labeling & Perfusion & Maze training & Maze probe \\
\hline Anterograde tracer $(1 a-d)$ & $\begin{array}{l}\text { Unilateral EC tracer } \\
\text { infusion }\end{array}$ & & $7 \mathrm{~d}$ after infusion & & \\
\hline S-induced Fos $(1 e-h)$ & Unilateral ECS & & $1 \mathrm{~h}$ after $\mathrm{S}$ & & \\
\hline S-induced DG survival $(3 d, e)$ & Unilateral ECS, NS & BrdU 1,10, or $30 \mathrm{~d}$ before $S$ & 3 weeks after $S$ & & \\
\hline S-induced SVZ proliferation $(4 a-c)$ & Unilateral ECS, NS & BrdU $3 \mathrm{~d}$ after $S$ & $24 \mathrm{~h}$ after BrdU & & \\
\hline Retroviral labeling of DGCs (5) & Unilateral ECS, NS & $\begin{array}{l}\text { Bilateral DG retrovirus infu- } \\
\text { sion } 3 \mathrm{~d} \text { after } S\end{array}$ & 1 or 6 weeks after infusion & & \\
\hline $\begin{array}{l}\text { S-induced DG neurogenesis ( } 3 a-c) \\
\text { and DGC integration (6) }\end{array}$ & Unilateral ECS, NS & IdU 3-5 d, CldU 7-9 d after S & $\sim 10$ weeks after XdU & $\begin{array}{l}\text { Standard training } \sim 1 \text { or } 6 \\
\text { weeks after } X \mathrm{dU} U\end{array}$ & $\sim 10$ weeks after $S$ \\
\hline S effects on spatial memory $(7,8)$ & Bilateral EC S, NS & & & $\begin{array}{l}\text { Undertraining } 1.5 \text { or } 6.5 \text { weeks } \\
\text { after, or } 1 \mathrm{~d} \text { before } S\end{array}$ & $1 \mathrm{~h}$ after training or 1.5 weeks after $S$ \\
\hline
\end{tabular}

NS, Nonstimulation; S, stimulation.

stimulation (50 $\mu \mathrm{A}$ for $1 \mathrm{~h})$. Mice were perfused 1 or 6 weeks ( $n=3$ each) later.

The long-term survival of stimulation-induced newly born neurons and their integration rate into spatial memory networks, relative to similarly aged cells, was assessed after unilateral EC stimulation (50 $\mu \mathrm{A}$ for $1 \mathrm{~h}$ ). Mice received IdU injections during the period of stimulationinduced increased proliferation (postoperative days 3-5) and CldU injections during a similar period of baseline proliferation (postoperative days 7-9). Water-maze standard training occurred 6 weeks $(n=17$, includes 5 mice used for NeuN phenotype experiment) or 1 week $(n=$ 15) later, followed by probe testing 4 or 9 weeks later, respectively. Mice were perfused $90 \mathrm{~min}$ after probe testing.

EC stimulation-induced changes in spatial memory performance were assessed in bilaterally stimulated ( $50 \mu \mathrm{A}$ for $1 \mathrm{~h}, n=25$ ) or nonstimulated $(n=26)$ mice who underwent water maze training (undertraining protocol) 6.5 weeks later and subsequent probe testing $1 \mathrm{~h}$ after the last training trial. In a second experiment, stimulated $(n=25)$ and nonstimulated $(n=26)$ mice were trained (undertraining protocol) 1.5 weeks after surgery. In the third experiment, mice were first trained (undertraining protocol), followed $1 \mathrm{~d}$ later by stimulation $(n=24)$ or nonstimulation $(n=24)$ surgery and subsequent probe testing 1 week later. In the final experiment, animals received V or TMZ injections followed on the next day by bilateral stimulation (V-S, $n=24$; TMZ-S, $n=24$ ) or nonstimulation (V-NS, $n=17$; TMZ-NS, $n=17$ ). Six and a half weeks after surgery, animals were trained in the water maze (undertraining protocol) and received a probe test $1 \mathrm{~h}$ after the last training trial. To assess the proportion of stimulation-induced newly born cells adopting a neuronal phenotype, BrdU was given intraperitoneally at a dose of $50 \mathrm{mg} / \mathrm{kg}$ per injection three times per day ( $8 \mathrm{~h}$ apart) for 3 consecutive days to subsets of animals in each group: on postoperative days 3-5 (V-S, $n=8$; TMZ-S, $n=5$; V-NS, $n=8$; TMZ-NS, $n=5$ ) during the period of stimulation-induced increased proliferation or for $3 \mathrm{~d}$ after the completion of water-maze testing (V-S, $n=5$; TMZ-S, $n=7$; V-NS, $n=7$; TMZ-NS, $n=5) \sim 7$ weeks postoperatively.

Statistical analyses. Behavioral (excluding swim strategy) and cell count (excluding scatter plots) data are presented as mean \pm SEM and were evaluated using ANOVAs or $t$ tests (two-tailed). Duncan's multiple range test was used for post hoc analyses of significant main effects or interactions when appropriate. Swim strategy frequencies were compared across trials within groups using Friedman's test and over all trials between groups using Pearson's $\chi^{2}$ test for independence. Scatter plot data were analyzed using Pearson's correlation.

\section{Results}

Entorhinal cortex projects ipsilaterally to the DG

The EC provides the main afferent input to the DG (Amaral and Lavenex, 2007). Because neurogenesis is regulated by neural ac- tivity (Zhao et al., 2008), stimulation of these afferents provides a direct method to increase adult neurogenesis. To characterize this projection, we first injected a fluorescent, anterograde tracer into the EC (Fig. 1a). Our injection site was centered on the junction of the ventral intermediate, medial, and caudal entorhinal fields (van Groen, 2001) (Fig. 1b). Viewed in horizontal sections, labeled axons extended anteromedially from the injection site. They coursed through the angular bundle region to the subiculum, past CA1-CA3, and around the hippocampal fissure via the stratum lacunosum moleculare, eventually reaching the molecular layer of the DG. Focal dilations and club-shaped terminals were evident along this path and were especially dense in the outer two-thirds of the molecular layer, a pattern that is consistent with terminal labeling of fibers from both the medial and lateral perforant paths (Amaral and Lavenex, 2007). Fibers also coursed along the alveus, consistent with direct alvear projections to the subiculum and CA1 (Amaral and Lavenex, 2007). In addition, projections also reached the presubiculum and, to a lesser extent, the parasubiculum (van Groen and Wyss, 1990). Labeling was virtually restricted to the side ipsilateral to the injection site, consistent with previous reports that the EC projection to the DG is predominantly ipsilateral (van Groen et al., 2002, 2003). Furthermore, terminal labeling was densest in the middle and posterior regions of the DG (Fig. $1 c, d$ ), consistent with known topographical organization of EC-DG connections (van Groen et al., 2002, 2003).

We next asked whether stimulation of the EC would increase activity in the DG. To do this, we lowered an electrode into the same EC site and delivered high-frequency stimulation for $1 \mathrm{~h}$ under chloral hydrate anesthesia. Our stimulation parameters $(50 \mu \mathrm{A}, 90 \mu$ s pulse width delivered at $130 \mathrm{~Hz}$ ) were chosen to model those used for high-frequency DBS clinically (Volkmann et al., 2006). Sixty minutes after the completion of stimulation, we assessed expression of the activity-regulated gene c-Fos (Fig. 1e). Consistent with the tracing experiment, Fos expression was elevated in the DG granule cell layer, suggesting that stimulation led to sustained activation of DGCs. The increase in Fos expression was limited to the side ipsilateral to the site of stimulation (Fig. 1f,g), consistent with the finding that the EC-DG projection is predominantly unilateral. Moreover, increased Fos expression was essentially confined to the middle and posterior portions of the DG, a pattern that matches the topographical organization of 
a

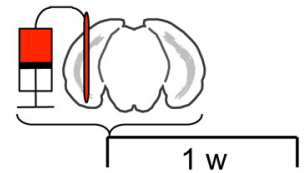

b

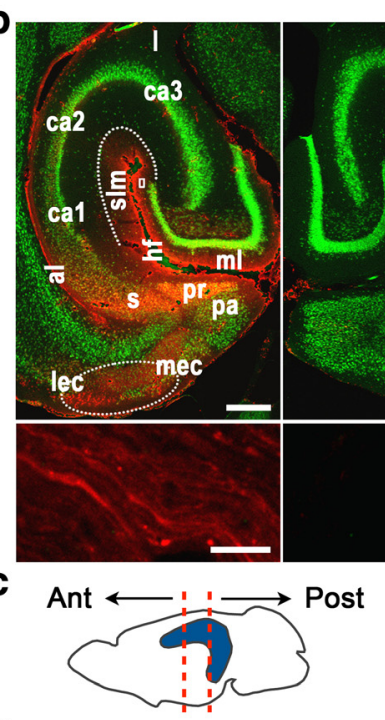

d
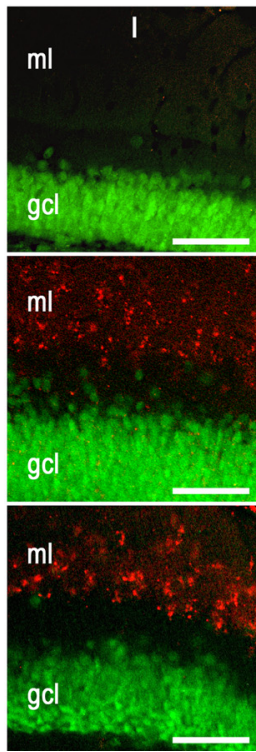

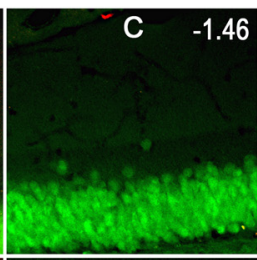

$-2.46$
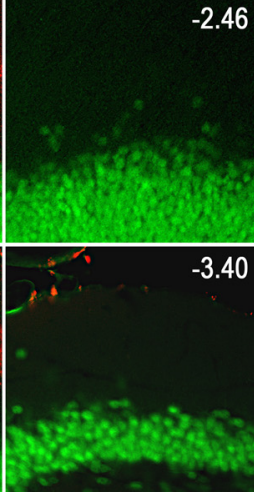

e

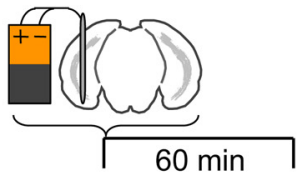

f

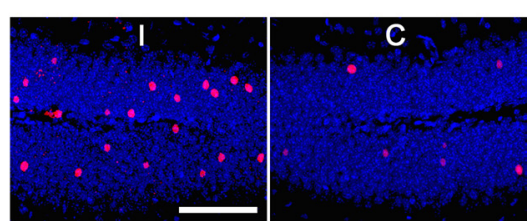

g

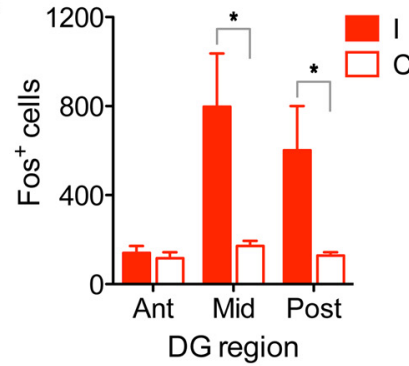

$\mathbf{h}$

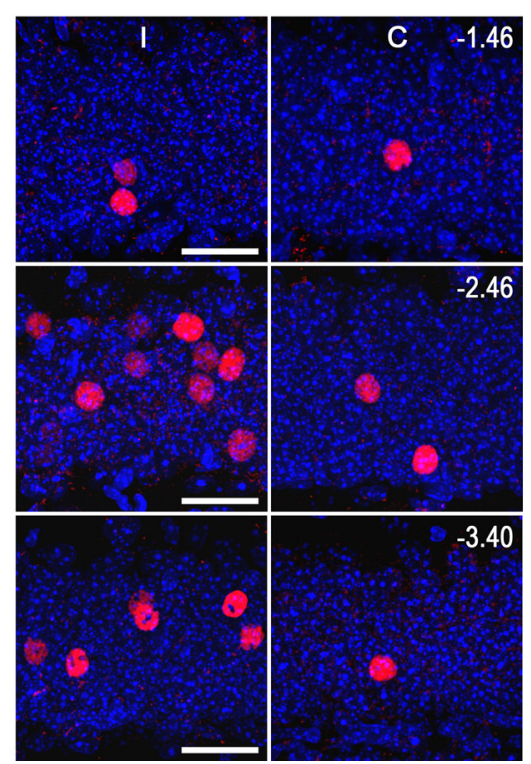

Figure 1. Entorhinal cortex projects ipsilaterally to the DG. $\boldsymbol{a}$, Tracer distribution was examined $7 \mathrm{~d}$ after unilateral EC target injection $(n=5)$. $\boldsymbol{b}$, NeuN-stained (green) horizontal section (top 2 panels; scale bar, $200 \mu \mathrm{m}$ ) showing tracer (red) distribution ipsilateral (I, left panels) and contralateral (C, right panels) to EC target (dotted oval) injection (al, alveus; ca1-3, cornu ammonis regions 1-3; hf, hippocampal fissure; lec, lateral EC; mec, medial EC; ml, molecular layer; pa, parasubiculum; pr, presubiculum; $\mathrm{s}$, subiculum; $s \mathrm{Im}$, stratum lacunosum moleculare). Squares in top panels indicate perforant path regions magnified in corresponding bottom panels (scale bar, $5 \mu \mathrm{m}$ ). c, Sagittal schematic indicating anterior (Ant), middle, and posterior (Post) thirds of DG. $\boldsymbol{d}$, Representative DG coronal sections ipsilateral (I, left panels) and contralateral (C, right panels) to tracer injection. Molecular layer afferents were labeled in the middle and posterior (bottom 2 panels) regions of the ipsilateral DG ( $g c l$, granule cell layer; numbers indicate anteroposterior distance to bregma; scale bar, $50 \mu \mathrm{m}$ ). e, Fos expression was examined $1 \mathrm{~h}$ after unilateral stimulation $(n=6) . f$, DAPI (blue)-stained coronal section showing DG Fos (red) expression ipsilateral (I, left panel) and contralateral (C, right panel) to stimulation (scale bar, $50 \mu \mathrm{m}$ ). $\boldsymbol{g}$, Fos expression increased in middle (Mid) and posterior (Post) regions of the ipsilateral (I), but not contralateral (C), DG. $\boldsymbol{h}$, Representative coronal sections ipsilateral (I, left panels) and contralateral (C, right panels) to stimulation from anterior (top panels), middle (middle panels), and posterior (bottom panels) DG regions (as per schematic in $c$; scale bar, $20 \mu \mathrm{m}){ }^{*} p<0.05$.

terminal labeling after injection of anterograde tracer into the same site (Fig. 1g,h). An ANOVA with Side (ipsilateral, contralateral) and Region (anterior, middle, posterior) as within-subject factors supported the anatomical specificity of these effects. Most critically, there was a significant Side $X$ Region interaction $\left(F_{(2,10)}=6.13, p<\right.$ $0.05)$, reflecting more pronounced changes in Fos expression in the ipsilateral middle and posterior regions of the DG (Duncan's post hoc tests comparing ipsilateral and contralateral sides, $p$ values $<0.05$ ).

\section{EC stimulation transiently increases adult neurogenesis in the DG}

We next asked whether stimulation of the EC would increase proliferative activity in the DG. To do this, mice were injected with the proliferation marker BrdU, 1, 3, 5 , or $7 \mathrm{~d}$ after stimulation of the EC. Twenty-four hours later, BrdU incorporation was quantified in the DG (Fig. $2 a$ ). EC stimulation increased the number of BrdU-labeled cells (Fig. 2b,c). An ANOVA, with Side (ipsilateral, contralateral) as a within-subjects variable and Stimulation (stimulated, nonstimulated) and Day (1, $3,5,7)$ as between-subject variables, revealed that these increases were anatomically specific and delay dependent (significant three-way Side $\times$ Stimulation $\times$ Day interaction, $\left.F_{(3,28)}=3.90, p<0.05\right)$ : increased BrdU incorporation was limited to the ipsilateral side and to groups injected with BrdU 3-5 d after stimulation (Duncan's post hoc tests, $p$ values $<0.05)$. These results indicate that the pro-proliferative effects of EC stimulation are not immediate but rather emerge after a few days. In addition, contralateral to the electrode site, $\mathrm{BrdU}$ incorporation was equivalent in both stimulated and nonstimulated mice (Duncan's post hoc tests, $p$ values $>0.05$ ). This indicates that stimulation does not modulate proliferation contralateral to the electrode site, and therefore the contralateral side may be used as a within-animal control for basal rates of proliferation after unilateral stimulation of the EC. Finally, to explore the topographical specificity of the stimulation-induced changes in proliferation in detail, we divided the DG into equal thirds from the anterior-to-posterior pole. In the $3 \mathrm{~d}$ group, we found that increased proliferation was confined to the middle and posterior ipsilateral DG (Fig. $2 d$; Side $\times$ Region ANOVA, significant interaction, $F_{(2,10)}=7.73, p<0.01$; Duncan's post hoc tests comparing ipsilateral and contralateral sides, $p$ values $<0.05)$. This topographical organization is consistent with the tracing and immediate early gene analyses.

We next evaluated how stimulation duration and intensity impacts DG proliferation. First, to examine the impact of duration, mice were stimulated for 30,60 , or $120 \mathrm{~min}$ using the same stimulation parameters as before. They were then injected with BrdU $3 \mathrm{~d}$ later, and, as previously, BrdU incorporation was assessed $24 \mathrm{~h}$ later. 
Increasing stimulation duration increased proliferation ipsilateral to the electrode site (Fig. $2 e$; Side $\times$ Duration ANOVA, significant interaction, $F_{(2,11)}=5.39, p<$ 0.05): there were more BrdU-labeled cells after $60 \mathrm{~min}$ (compared with $30 \mathrm{~min}$ ) stimulation (Duncan's post hoc test, comparing 30 and $60 \mathrm{~min}$ on ipsilateral side; $p<0.01$ ) but no additional benefit of further increasing stimulation duration to 120 min (Duncan's post hoc test, comparing 60 and 120 min on ipsilateral side; $p=$ 0.42 ). Second, to examine the impact of current intensity on proliferation, mice received 50,250 , or $500 \mu \mathrm{A}$ of stimulation for $60 \mathrm{~min}$. They were then injected with BrdU 3 d later, and BrdU incorporation was assessed $24 \mathrm{~h}$ later. Although stimulation-induced changes in granule cell layer/subgranular zone proliferation were limited to the ipsilateral side, there were no effects of stimulation intensity (Fig. 2f; Side $\times$ Intensity ANOVA, significant main effect of Side only, $F_{(1,11)}=$ 45.76, $p<0.01$ ), suggesting that there might be a ceiling on these stimulationinduced changes in proliferation. Indeed, at 250 and $500 \mu \mathrm{A}$, BrdU-labeled cells were additionally found in the hilus and molecular layer, suggesting that highintensity stimulation may additionally promote proliferation of non-neuronal cell types (Madsen et al., 2005) and/or aberrant neurogenesis analogous to that seen after seizures (Parent et al., 1997, 2006).

Under basal conditions, $\sim 70-90 \%$ of cells generated in the adult subgranular zone differentiate into neurons (Brandt et al., 2003). To evaluate whether a similar proportion of cells differentiate into neurons after stimulation of the EC, mice were treated with equimolar doses of IdU and then CldU, two chemically related thymidine analogs. IdU and CldU are recognized by different antibodies and therefore may be used to label separate cohorts of cells in the same animal (Vega and Peterson, 2005; Stone et al., 2010; Tronel et al., 2010). In this experiment, IdU injections occurred 3-5 d after stimulation, a poststimulation time point corresponding to the peak in stimulation-induced changes in proliferation, and CldU injections occurred 7-9 d after stimulation, a poststimulation time point when proliferation rates have returned to baseline (Fig. 3a). Ten weeks later, there were no differences in the proportion of IdU- and CldU-labeled cells expressing the neuronal marker NeuN, either ipsilateral or contralateral to the electrode site (Fig. 3b,c; Side $\times$ Thymidine Analog ANOVA, main effects and interaction $p$ values $>0.05$ ), indicating that stimulation did not alter the fate of newly generated cells. Importantly, the number of IdU-labeled cells was increased on the side ipsilateral to the stimulation site $(298.40 \pm 14.94)$ compared with IdU-labeled cells in the contralateral DG $(186.72 \pm 11.74)$ and CldU-labeled cells in the ipsilateral (192.04 \pm 12.29$)$ and contralateral $(186.53 \pm 11.55) \mathrm{DG}$. The magnitude of the relative increase after this extended survival period ( $\sim 10$ weeks) is comparable with that observed after a short survival period ( $1 \mathrm{~d}$; Fig. $2 c)$, suggesting that cells produced as a consequence of stimulation survive for at least several weeks.

We found that stimulation of the EC transiently increased the future production of neurons in the DG. To evaluate whether the same stimulation promotes survival of existing, adult-generated cells, we next treated mice with the proliferation marker BrdU and then stimulated 1, 10, or 30 d later (Fig. $3 d$ ). The numbers of BrdU-labeled cells were increased ipsilaterally (vs contralaterally) in the $10 \mathrm{~d}$ (planned comparison, paired $t$ test, $t_{(8)}=9.31, p<$ 0.01 ) group but not in the $1 \mathrm{~d}$ (planned comparison, paired $t$ test, $t_{(8)}=0.43, p=0.68$ ) or $30 \mathrm{~d}$ (planned comparison, paired $t$ test, $t_{(8)}=0.38, p=0.72$ ) groups (Fig. $3 e$; one-way ANOVA, significant effect of Group, $F_{(2,21)}=5.46, p<0.05$; Duncan's post hoc tests, $p$ values $<0.05)$. Consistent with previous reports (BruelJungerman et al., 2006; Kitamura et al., 2010), these data indicate that EC stimulation has a modest pro-survival effect on existing adult-generated cells in the hippocampus. That these effects were specific to the $10 \mathrm{~d}$ group is consistent with the idea that 1-to 3 -week-old neurons are especially sensitive to factors that either promote (e.g., environmental enrichment) or reduce (e.g., stress) survival (Zhao et al., 2008). 
a

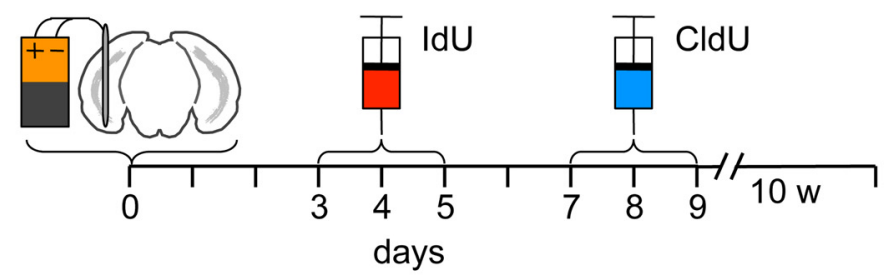

b
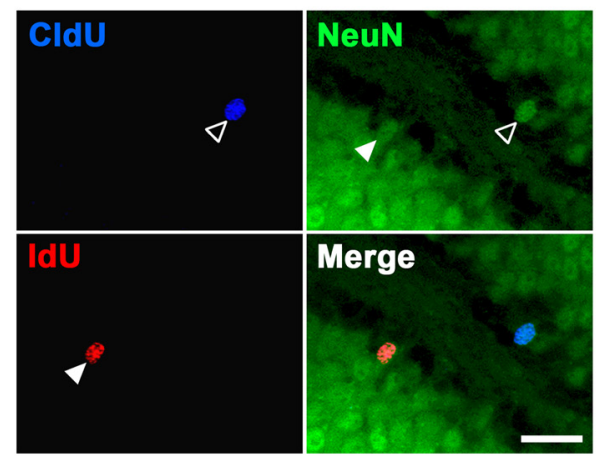

d

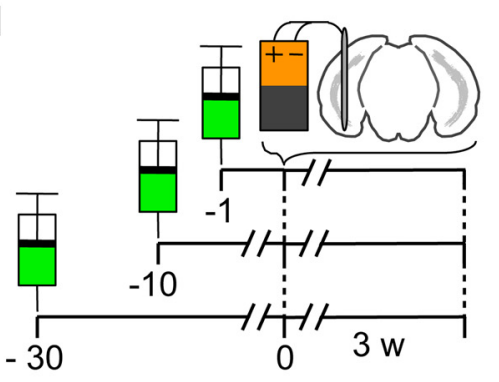

days

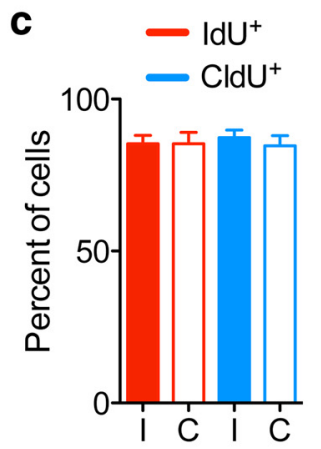

e 350

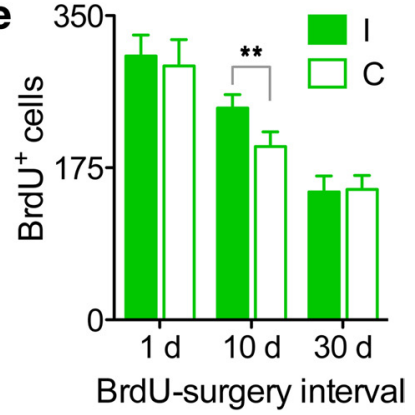

Figure 3. Survival and differentiation of stimulation-induced neurons. $\boldsymbol{a}$, After unilateral stimulation $(n=5)$, mice were injected with IdU (during the period of increased proliferation) and CIdU (once proliferation returned to baseline). $\boldsymbol{b}$, Representative confocal image of IdU ${ }^{+}$and CldU ${ }^{+}$DG cells colabeled with NeuN (scale bar, $20 \mu \mathrm{m}$ ), ipsilateral to stimulation. c, Similar proportions of IdU ${ }^{+}$and $\mathrm{CldU}^{+}$cells were NeuN ${ }^{+}$ipsilateral (I) and contralateral (C) to electrode site. $d$, Separate groups of mice were injected with BrdU at different delays before stimulation ( $n=8$ per group). $\boldsymbol{e}$, There were more BrdU ${ }^{+}$cells ipsilateral to the stimulation site in the mice treated with BrdU $10 \mathrm{~d}$ before surgery. ${ }^{* *} p<0.01$.

\section{Specificity of stimulation-induced increase in proliferation}

In the above experiments, we identified a set of conditions whereby EC stimulation produces an approximate 1.5-fold to 2 -fold increase in adult neurogenesis. We next explored the specificity of the effects of stimulation.

First, the subgranular zone of the hippocampus is one of two major neurogenic regions in the adult brain. Does stimulation of the EC additionally regulate neurogenesis in the subventricular zone? To address this, additional groups of mice were treated with BrdU $3 \mathrm{~d}$ after stimulation of the EC (Fig. 4a). Twenty-four $\mathrm{h}$ later, BrdU-labeled cells were evident in the subependymal region of the subventricular zone (Fig. $4 b$ ). There were similar numbers of BrdU-labeled cells in both the stimulation and nonstimulation groups, and no additional differences between the ipsilateral and contralateral sides (Fig. $4 c$; Side $\times$ Stimulation ANOVA, main effects and interaction $p$ values $>0.05$ ), suggesting that EC stimulation specifically regulates hippocampal and not olfactory adult neurogenesis. Similarly, the pro-neurogenic effects after stimulation of another limbic target, the anterior thalamic nucleus, are limited to the DG (Encinas et al., 2011). This contrasts with the biregional neurogenic effect seen after generalized neural stimulation by seizures (Parent et al., 2002).

Second, high-intensity, generalized electrical activation of the brain (e.g., electroconvulsive shock, seizures) also increases adult neurogenesis in the DG (Madsen et al., 2000; Parent et al., 2002;
Ma et al., 2009). Using our lower-current intensity, we next evaluated whether localized stimulation elsewhere in the brain might increase adult neurogenesis in the DG. To do this, we targeted the fourth and fifth cerebellar lobules, regions that do not send direct afferent input to the DG (Fig. $4 d$ ). BrdU incorporation $3 \mathrm{~d}$ after stimulation was unaltered by cerebellum stimulation (compared with a nonstimulated control group) (Fig. $4 e$; Side $\times$ Stimulation ANOVA, main effects and interaction $p$ values $>0.05)$, suggesting that low-intensity stimulation of non-afferent sites does not regulate adult neurogenesis in the DG.

Third, it is possible that stimulation of the EC might induce cell death in the DG, leading to a compensatory increase in adult neurogenesis (Gould and Tanapat, 1997). Therefore, to evaluate the possibility that changes in neurogenesis are secondary to altered levels of DGC death, we additionally stained tissue for TUNEL, a marker of programmed cell death, in stimulated or control mice 2, 4, 6, or $8 \mathrm{~d}$ after stimulation. Although there were regional differences in TUNEL counts, with levels highest in the granule cell layer and lowest in the hilus, no such differences were observed between the ipsilateral and contralateral sides, stimulated and nonstimulated groups, or days after stimulation (Fig. $4 f-h$; Side $\times$ Region $\times$ Stimulation $\times$ Delay ANOVA, significant main effect of Region only, $F_{(2,28)}=$ 193.94, $p<0.01)$. Together, these data suggest that our low-intensity EC stimulation does not induce apoptotic cell death in the DG and therefore exclude the possibility that changes in levels of adult neurogenesis are secondary to stimulation-induced cell death.

\section{After stimulation, new neurons mature normally}

We found that EC stimulation increases adult neurogenesis in the DG. Because these newly generated neurons survive several weeks, this raises the possibility that they become functionally integrated into hippocampal circuits. To evaluate this possibility, we used a retroviral vector strategy to label dividing neural progenitors and their progeny with GFP (Tashiro et al., 2006). Because GFP is expressed throughout the cell body and processes, using this strategy, it is possible to track morphological changes in newborn cells at different stages after stimulation. Accordingly, mice received unilateral stimulation and then, $3 \mathrm{~d}$ later, bilateral infusion of retrovirus encoding GFP into the DG. This $3 \mathrm{~d}$ poststimulation time point corresponds to the peak of stimulation-induced changes in proliferation. Mice were killed either 1 or 6 weeks later.

In the 1 week group (Fig. $5 a$ ), there were approximately twice as many GFP-labeled cells per section on the ipsilateral (27.09 \pm $5.20)$ versus contralateral $(13.95 \pm 2.38)$ side to the stimulation site (Fig. $5 b$; paired $t$ test, $t_{(2)}=4.64, p<0.05$ ), reflecting stimulation-induced changes in proliferation. Both the magnitude and the anatomical specificity of this increase replicate our 
BrdU incorporation data. Most of GFPlabeled cells were located in the subgranular zone or innermost part of the granule cell layer and had short, aspiny, processes running either parallel to or, occasionally, perpendicular to the granule cell layer. In rare instances, these perpendicular processes extended through the granule cell layer (but never into the molecular layer). No axon fibers were visible in CA3. Consistent with previous reports (Zhao et al., 2006; Jessberger et al., 2007), the morphology of these GFP-labeled cells resembled immature neurons. A small minority of cells $(\sim 2 \%)$ were ectopically located in the hilus or deep within the granule cell layer. Importantly, the frequency of these ectopically located cells was similar ipsilateral $(1.77 \%)$ and contralateral (1.74\%) to the electrode site (paired $t$ test, $t_{(2)}=$ $0.05, p=0.96$ ), indicating that stimulation did not alter the normal migration and early stages of integration of adultgenerated neurons in the DG.

Six weeks after infection (Fig. $5 c$ ), there were many GFP-labeled cells resembling mature neurons. As before, there were approximately twice as many GFP-labeled cells on the ipsilateral $(4.47 \pm 0.19)$ versus contralateral $(2.11 \pm 0.17)$ side to the stimulation site (Fig. $5 d$; paired $t$ test, $\left.t_{(2)}=16.28, p<0.01\right)$, suggesting that cells, produced as a consequence of stimulation, survive for at least several weeks. The vast majority of GFP-labeled cells were located in the subgranular zone or innermost part of the granule cell layer. Typically, dendritic processes extended through the granule cell layer with minimal or no branching but arborized extensively in the molecular layer. Furthermore, there were abundant spines on these apical dendritic processes, especially within the molecular layer, suggesting that these newly generated neurons had become synaptically integrated into DG circuitry. GFPlabeled axonal fibers were also present in the CA3 region, consistent with previous reports suggesting that, by 6 weeks of age, adult-generated neurons have established efferent connections with CA3 (Zhao et al., 2006; Toni et al., 2008). Most importantly, apical dendrite branch morphology was similar on both the ipsilateral and contralateral sides to the electrode site, with equivalent total dendrite length (Fig. $5 e$; paired $t$ test, $t_{(2)}=0.50, p=$ 0.67 ) and nodes per neuron (Fig. $5 f$; paired $t$ test, $t_{(2)}=0.09$, $p=0.94)$. Furthermore, spine density on apical dendritic branches was similar both ipsilateral and contralateral to the electrode site (Fig. $5 g$; Side $\times$ Branch Order ANOVA, significant main effect of Branch Order only, $F_{(4,2)}=34.24, p<0.01$ ). Together, these analyses suggest that stimulation promotes neurogenesis and that neurons produced as a consequence of stimulation acquire normal morphology and likely establish normal afferent and efferent connections. Similar to the 1 week postinfection group, a small minority of GFP-labeled cells were ectopically located. The low incidence is consistent with previous reports (Parent et al., 1997; Jessberger et al., 2007), and, impor-
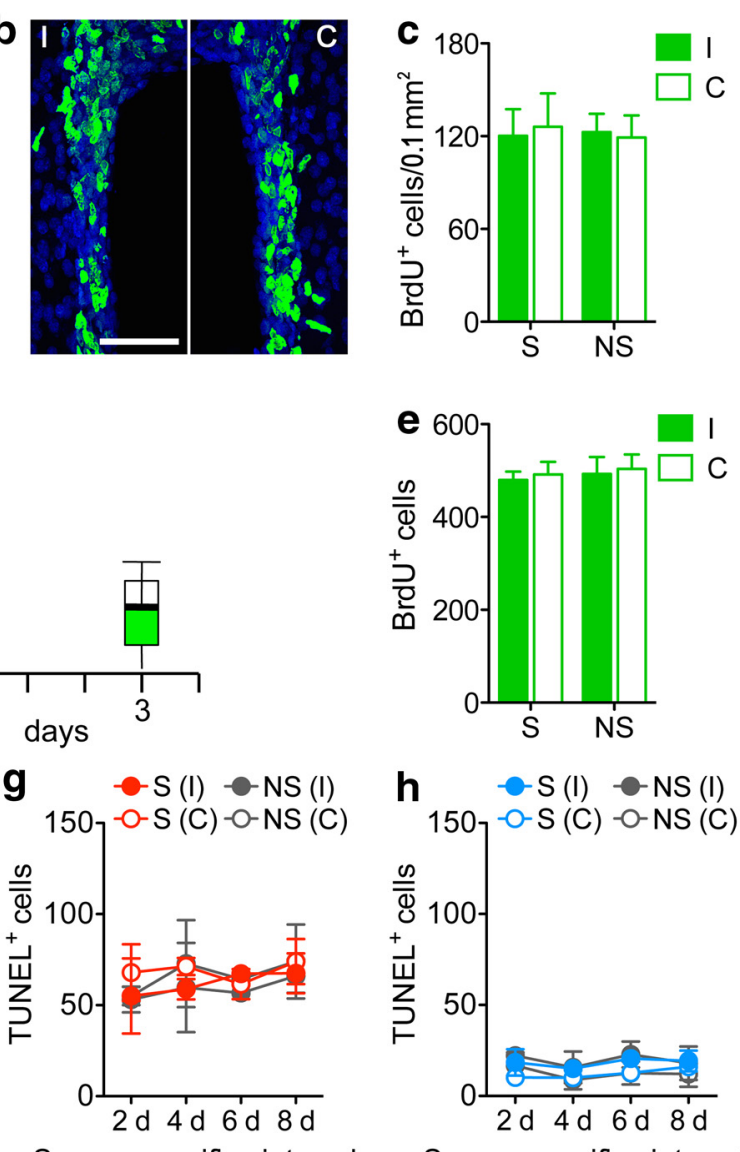

Surgery-sacrifice interval

Figure 4. Specificity of EC stimulation effects on hippocampal neurogenesis. $\boldsymbol{a}$, Mice were injected with BrdU 3 dafter unilateral stimulation or electrode insertion only ( $n=4$ per group). $\boldsymbol{b}, \mathrm{BrdU}^{+}$(green) cells in the subventricular zone (DAPI counterstain) 作 contralateral (C) SVZ. $\boldsymbol{d}$, In a separate experiment, mice were injected with BrdU $3 \mathrm{~d}$ after unilateral cerebellar stimulation or or nonstimulated (NS) mice in either the ipsilateral (I) or contralateral (C) DG. $\boldsymbol{f}-\boldsymbol{h}$, TUNEL counts were similar in the ipsilateral and contralateral granule cell layer $(\boldsymbol{f})$, molecular layer $(\boldsymbol{g})$, or hilus $(\boldsymbol{h})$ in stimulated (S) versus nonstimulated (NS) groups.

tantly, the frequency was similar on both the ipsilateral $(1.79 \%)$ and contralateral $(2.08 \%)$ sides (paired $t$ test, $t_{(2)}=0.19, p=$ 0.87 ), suggesting that stimulation does not promote the aberrant integration of adult-generated neurons into the DG.

\section{New neurons functionally integrate into hippocampal memory circuits}

The morphological analyses indicate that new neurons, generated as a consequence of stimulation, integrate into hippocampal circuitry. Using a water-maze task, we next asked whether, once integrated, these adult-generated neurons play a functional role in hippocampus-mediated spatial learning. To address this, mice were treated with equimolar doses of IdU and then CldU at different times after unilateral stimulation of the EC. As before, IdU injections occurred 3-5 d after stimulation (a time point corresponding to the peak in stimulation-induced changes in proliferation), and CldU injections occurred 7-9 d after stimulation (a time point when proliferation rates have returned to baseline). Six weeks later, mice were trained in the water maze, and then spatial memory was assessed in a probe test 4 weeks later (Fig. 6a). Both acquisition and expression of water-maze memories engage the DG, and engagement of dentate granule neurons after memory recall may be assessed by quantifying expression of activity- 
a

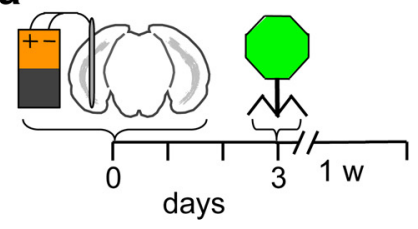

c

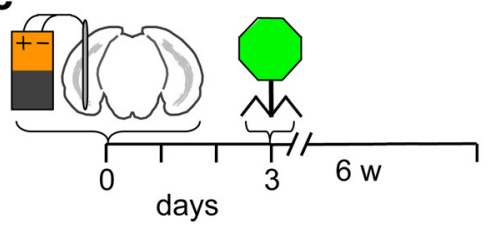

b

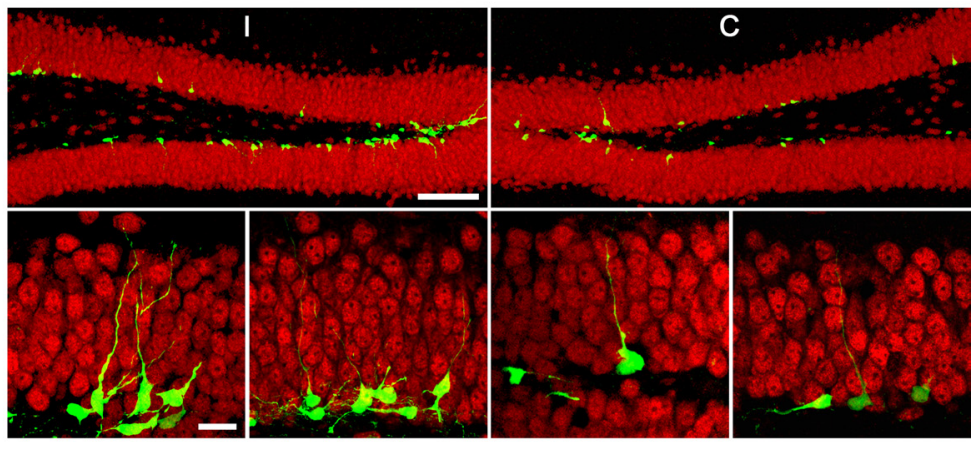

d

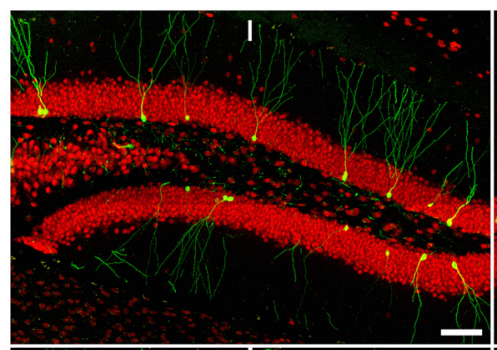

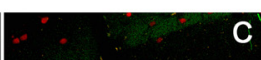

C.
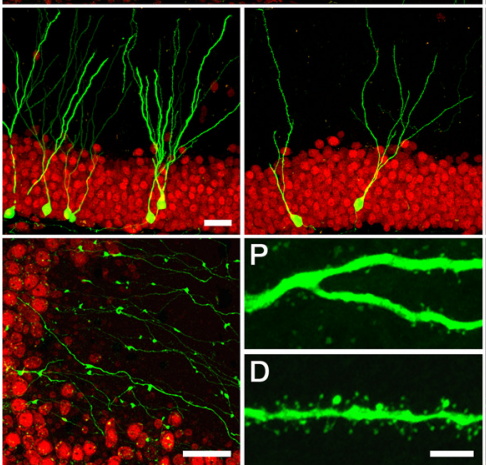

g

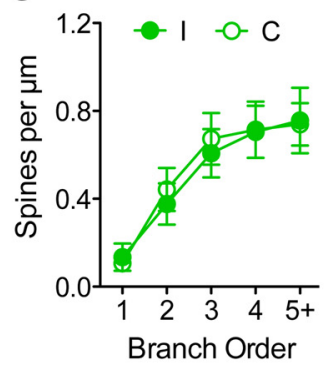

Branch Order f

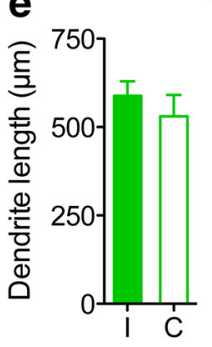

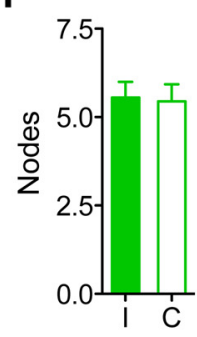

Figure 5. Morphology of adult-generated granule cells. $a$, Unilaterally stimulated mice received bilateral GFP-retrovirus injections into the DG $3 \mathrm{~d}$ after stimulation (during the period of increased proliferation) and were examined 1 week later $(n=3) \cdot \boldsymbol{b}$, Representative images ipsilateral (I, left 3 panels) and contralateral (C, right 3 panels) to stimulation at low (top panels; scale bar, $50 \mu \mathrm{m})$ and high (bottom panels; scale bar, $10 \mu \mathrm{m}$ ) magnification showing immature-appearing GFP ${ }^{+}$(green) neurons (red, NeuN counterstain). c, A separate group was examined 6 weeks after retroviral injections $(n=3)$. $\boldsymbol{d}$, Representative images ipsilateral (I, left 6 panels) and contralateral (C, right 6 panels) to stimulation. Low (top row; scale bar, $50 \mu \mathrm{m}$ ) and high (middle row; scale bar, $20 \mu \mathrm{m}$ ) magnification images show examples of mature-appearing neurons. GFP ${ }^{+}$axons and terminals were present bilaterally in CA3 (bottom row left panels; scale bar, $20 \mu \mathrm{m}$ ). High-magnification images (bottom row right panels; scale bar, $10 \mu \mathrm{m}$ ) show relatively aspiny proximal ( $\mathrm{P}$, top panels), and spiny distal (D, bottom panels) portions of apical dendrites. $\boldsymbol{e}-\boldsymbol{g}$, Similar total dendrite length $(\boldsymbol{e})$, average nodes per neuron $(\boldsymbol{f})$, and progressive increase in linear spine density by branch order $(\boldsymbol{g})$ ipsilateral versus contralateral to stimulation.

regulated genes such as c-Fos (Kee et al., 2007a). Therefore, should stimulation increase the pool of functional new neurons, then the proportion of adult-generated neurons included in the population of activated (Fos-labeled) neurons should increase. Such an increase should be specific to neurons generated $3-5 \mathrm{~d}$ after stimulation (i.e., IdU-labeled cells) and, because stimulation is unilateral, occur only on the side ipsilateral to the electrode site.

In the probe test, mice searched selectively, spending more time in the region of the pool that formerly contained the platform (Fig. $6 b$; paired $t$ test, $t_{(16)}=5.53, p<0.01$ ). Consistent with our previous experiments, stimulation of the EC led to an increase in adult neurogenesis. As expected, this increase was specific to the ipsilateral side and corresponded to new cells generated 3-5 d (i.e., IdU-labeled) after stimulation (Fig. $6 c, d$; Side $\times$ Thymidine Analog ANOVA, significant interaction, $F_{(1,16)}=$ 167.76, $p<0.01$; Duncan's post hoc tests, $p<0.01$ ). After the probe test, Fos expression was equivalent both ipsilateral and contralateral to the stimulation site, suggesting equivalent levels of activation (Fig. $6 e$; paired $t$ test, $t_{(16)}=0.98, p=0.34$ ). Within this population of Fos-labeled neurons, there were many IdU- or CldU-labeled cells. Most importantly, stimulation increased the proportion of adult-generated neurons included in this population of activated (Fos-labeled) neurons. Critically, this increase was specific to neurons generated 3-5 d after stimulation (i.e., IdU-labeled cells) and occurred only on the side ipsilateral to the electrode site (Fig. 6f; Side $\times$ Thymidine Analog ANOVA, significant interaction, $\left.F_{(1,16)}=11.33, p<0.01\right)$. Furthermore, this increase was proportional to the magnitude of the stimulationinduced increase in adult neurogenesis (Fig. $6 g ; r=0.58, p<0.05$ ). Therefore, these data suggest that EC stimulation increases the production of new neurons and that these new neurons assume functional roles in hippocampal circuits mediating memory.

The integration of adult-generated neurons into hippocampal memory circuits is maturation dependent, with new neurons not contributing in maximal numbers until they are $>5$ weeks of age (Kee et al., 2007b; Stone et al., 2010). Therefore, stimulationinduced increases in the contribution of adult-generated neurons should not occur if there is only a brief delay between stimulation and training. Accordingly, we repeated the above experiment but trained mice 1 week (rather than 6 weeks) after IdU/CldU injections (Fig. 6h). In the probe test 9 weeks later, mice searched selectively in the region of the pool formerly containing the plat- 
form (Fig. 6i; paired $t$ test, $t_{(14)}=8.16, p<$ $0.01)$. As before, stimulation of the EC led to an increase in adult neurogenesis. This increase was specific to the ipsilateral side and corresponded to new neurons generated 3-5 d (i.e., IdU-labeled) after stimulation (Fig. 6j; Side $\times$ Thymidine Analog ANOVA, significant interaction, $F_{(1,14)}=$ 33.48, $p<0.01$; Duncan's post hoc tests, $p<0.01)$. After the probe tests, Fos expression was equivalent both ipsilateral and contralateral to the stimulation site, suggesting equivalent levels of activation (Fig. $6 k$; paired $t$ test, $t_{(14)}=0.14, p=$ $0.89)$. However, this Fos-labeled population of dentate granule neurons contained few IdU- or CldU-labeled cells (Fig. 6l; Side $\times$ Thymidine Analog ANOVA, no significant interaction, $F_{(1,14)}=0.02, p=$ $0.89)$. Furthermore, there was no relationship between stimulation-induced increases in neurogenesis and the proportion of adult-generated neurons included in this population of activated (Fos-labeled) neurons (Fig. $6 m ; r=0.04, p=0.89$ ). These data are therefore consistent with the idea that 1-week-old adult-generated neurons are insufficiently mature to be integrated into hippocampal memory circuits (Kee et al., 2007b; Stone et al., 2010).

Together, these data suggest that stimulation increases the pool of functional newborn neurons. An ANOVA, conducted on data from both experiments, revealed a significant three-way Delay $\times$ Thymidine Analog $\times$ Side interaction $\left(F_{(1,30)}=4.79, p<0.05\right)$. This supports the conclusion that stimulation-induced increases in contribution of adult-generated neurons to water-maze memory are maturation dependent (they are observed only in the 6 week group), as well as anatomically selective (only occurring on the side ipsilateral to the electrode site) and temporally specific (corresponding to neurons generated 3-5 d after stimulation). Furthermore, the temporal relationships between stimulation, labeling (IdU and CldU injections), and testing were equivalent in both experiments. Therefore, because IdU- and CldU-labeled cells were equivalently aged at the time of testing, these data indicate that stimulation does not simply lower the threshold for activation (and/or Fos induction) of DGCs.

\section{EC stimulation facilitates spatial memory} formation in a delay-dependent manner We next asked whether stimulationinduced increases in neurogenesis would facilitate water-maze learning in adult mice. To maximize production of new neurons, mice received bilateral (rather a
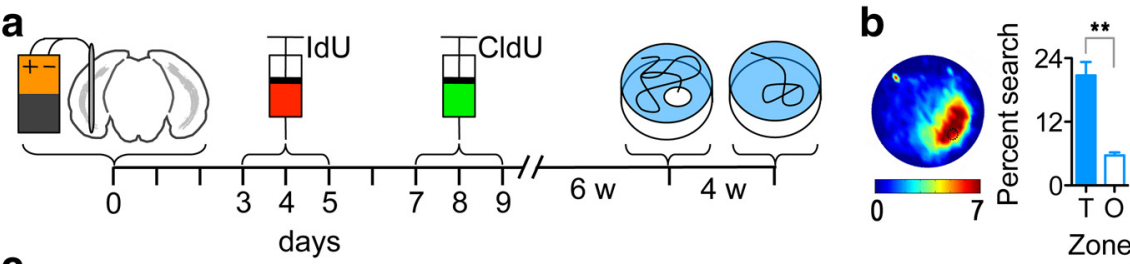

C

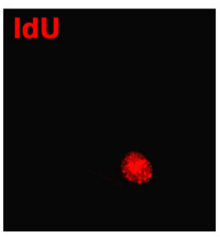

CldU
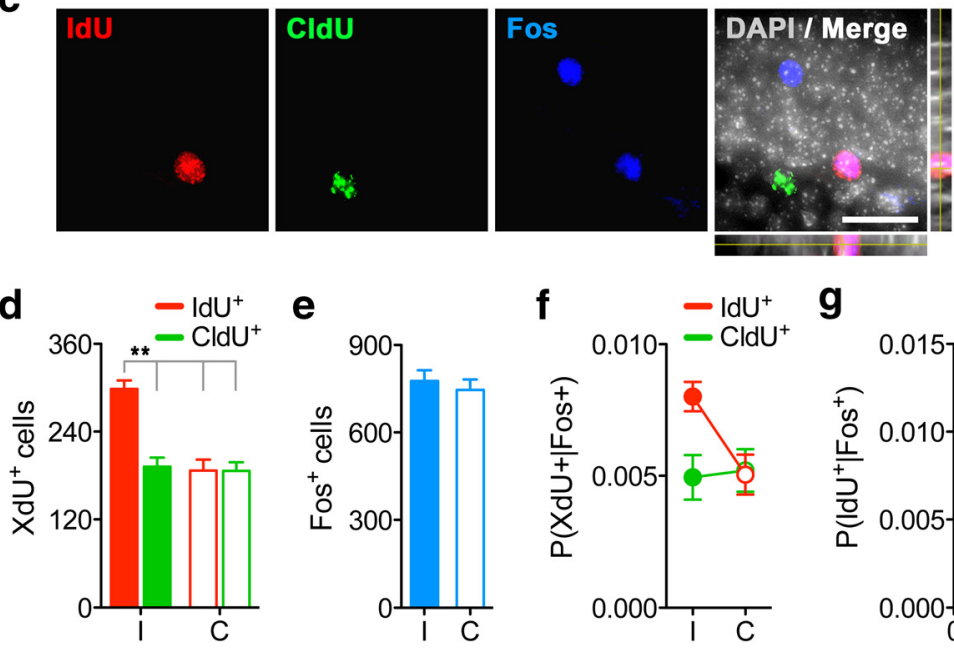

Zone

h
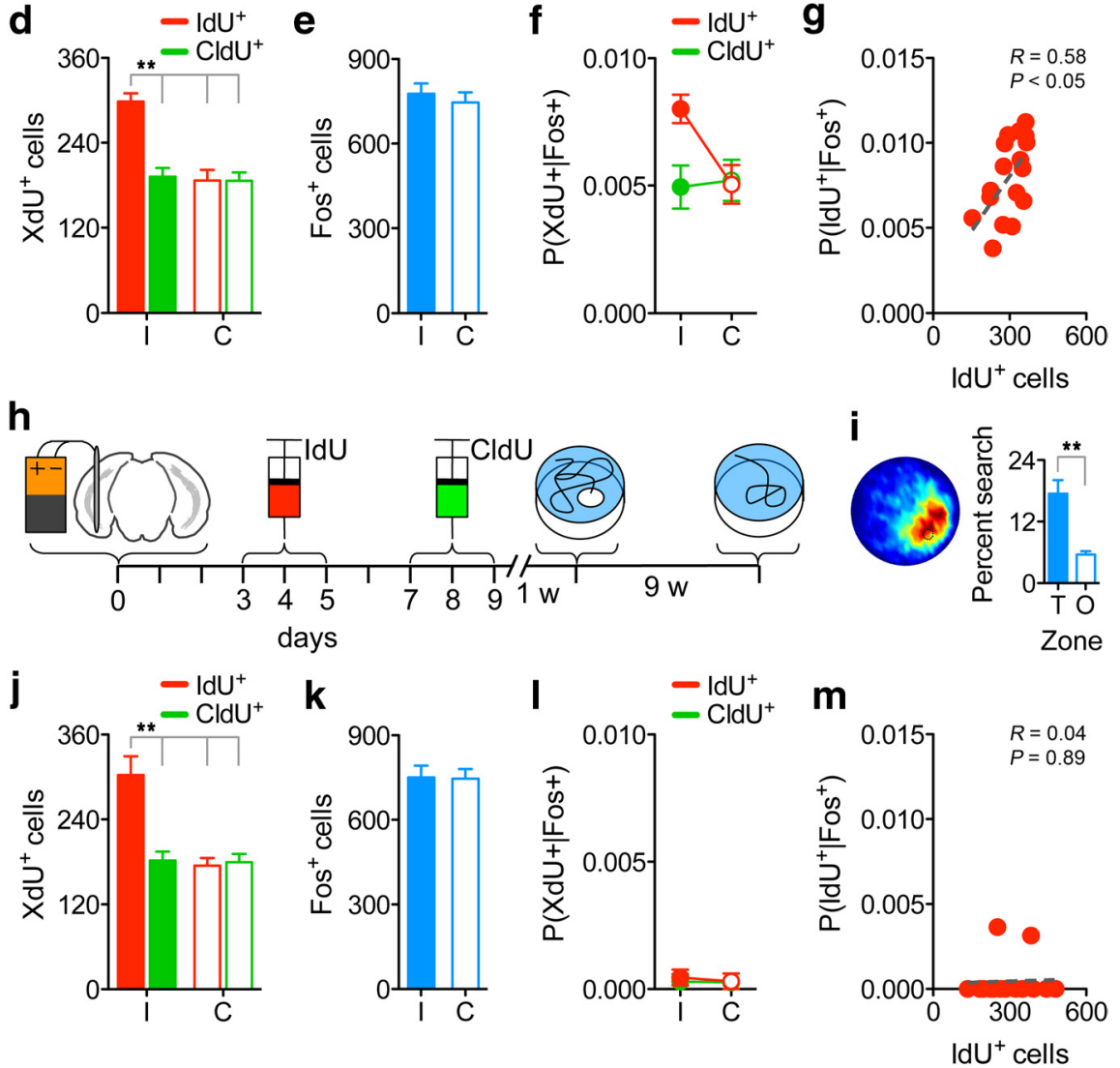

Figure 6. Functional integration of stimulation-induced neurons into hippocampal memory networks. $\boldsymbol{a}$, After unilateral stimulation, mice were injected with IdU during the period of increased proliferation and CIdU once proliferation returned to baseline $(n=17)$. Mice were trained 6 weeks after CIdU treatment, and spatial memory was assessed 4 weeks after the completion of training. $\boldsymbol{b}$, Mice searched selectively in the probe test. A density plot for grouped data (left), with accompanying color scale, represents the number of visits per mouse per $5 \times 5 \mathrm{~cm}$ area. Mice spent more time (right graph) searching the target zone $(T)$ compared with other $(0)$ zones. $c$, Representative confocal images of tissue after water-maze testing showing IdU ${ }^{+}$, $\mathrm{CldU}^{+}$, and Fos $^{+}$DGCs and a DAPI counterstained merge image with a representative IdU ${ }^{+} /$Fos $^{+}$colabeled cell (scale bar, $20 \mu \mathrm{m}$ ). $\boldsymbol{d}$, Numbers of IdU ${ }^{+}$and CldU ${ }^{+}$cells ipsilateral (I) versus contralateral (C) to stimulation site. e, Fos expression in the DG after probe testing was similar on both sides. $f$, After the probe test, the probability of Fos ${ }^{+}$cells being XdU ${ }^{+}$ipsilateral (I) and contralateral (C) to stimulation ( $y$-axis notation denotes the conditional probability of a cell being $X d U^{+}$given it is Fos ${ }^{+}$, or $P\left(X d U^{+} \mid F_{0 s}{ }^{+}\right)$). $\boldsymbol{g}$, As the number of $\mathrm{IdU}^{+}$cells increases, so does their contribution to the population of activated neurons (i.e., $\mathrm{P}\left(\mathrm{IdU}^{+} \mid \mathrm{Fos}^{+}\right)$). $\boldsymbol{h}$, A separate group of mice underwent identical treatment except were trained 1 week after CldU treatment and had spatial memory assessed 9 weeks after the completion of training $(n=15)$. $i$, Mice searched selectively in the probe test (left density plot), spending more time (right graph) searching the target zone compared with other zones. $j$, Numbers of IdU ${ }^{+}$and CldU $^{+}$cells ipsilateral (I) versus contralateral (C) to stimulation site. $\boldsymbol{k}$, Fos expression in the DG after probe testing was similar on both sides. $I$, The probability of Fos ${ }^{+}$cells being $\mathrm{XdU}^{+}$(or adult generated neurons) was equivalently low ipsilateral and contralateral to stimulation for IdU ${ }^{+}$and CldU ${ }^{+}$cells. $\boldsymbol{m}$, The stimulation-induced increase in the availability of adult-generated neurons did not produce a proportional increase in their contribution to the population of activated neurons, suggesting that neurons 1 week old at the time of training are not functionally integrated. ${ }^{* *} p<0.01$. 
a
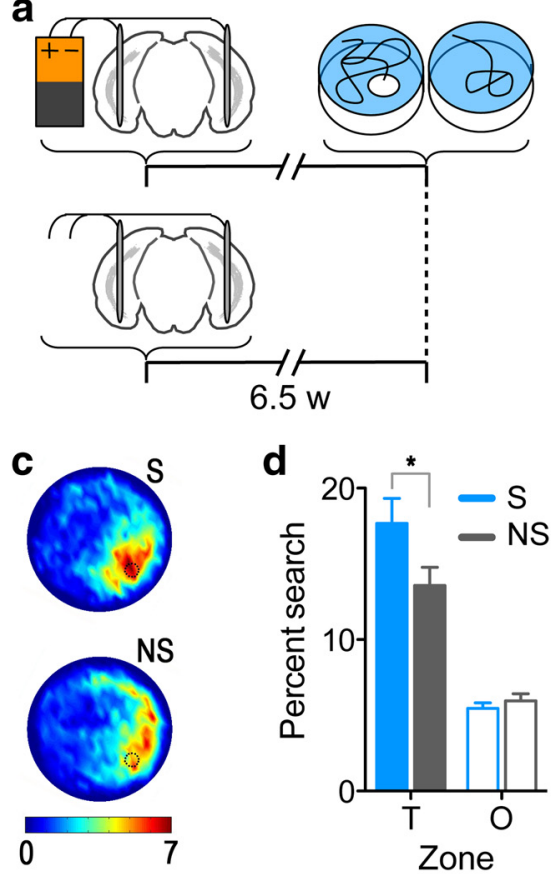

f Direct Focal
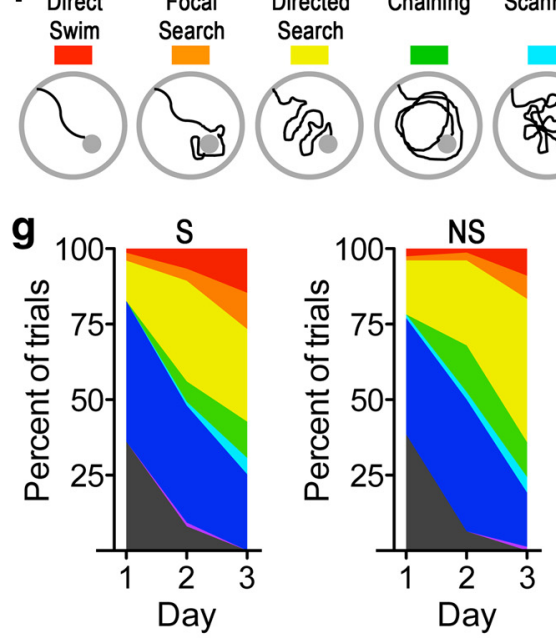

b

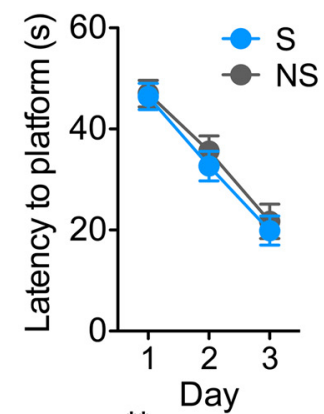

e

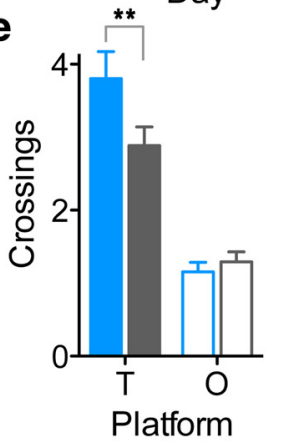

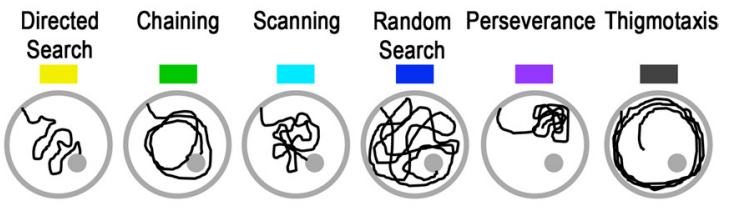

h

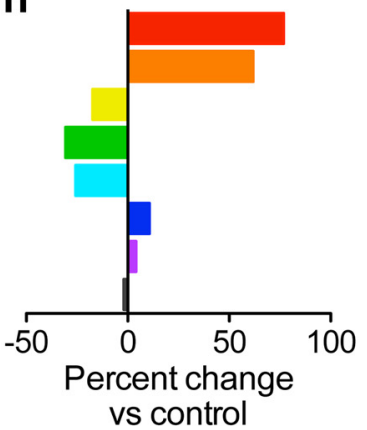

Figure 7. Stimulation-induced enhancement of spatial memory. $\boldsymbol{a}$, At 6.5 weeks after bilateral stimulation $(n=25)$ or electrode insertion only $(n=26)$, mice were trained in the water maze and spatial memory was assessed $1 \mathrm{~h}$ after the completion of training. $\boldsymbol{b}$, Latencies to reach the platform declined equivalently for stimulated (S) and nonstimulated (NS) mice during training. c, Mice searched selectively in the probe test. Density plots for grouped data, with accompanying color scale, represent the number of visits per mouse per $5 \times 5 \mathrm{~cm}$ area. $\boldsymbol{d}, \boldsymbol{e}$, Stimulated mice spent significantly more time searching the target zone $(\boldsymbol{d})$ and crossing the target platform location $(\boldsymbol{e})$ than nonstimulated mice. $\boldsymbol{f}$, Examples of search strategies with corresponding color labels. $\boldsymbol{g}$, Percentage of trials classified in each search strategy category across training days for stimulated (left graph) and nonstimulated (right graph) mice. $\boldsymbol{h}$, Percentage difference in the frequency of each search strategy in stimulated relative to nonstimulated mice, indicating significantly greater use of direct swim and focal search strategies in stimulated mice. ${ }^{*} p<0.05,{ }^{* *} p<0.01$.

than unilateral) stimulation of the EC and were trained in the water maze 6.5 weeks later. Control mice were treated identically except that no current was delivered (Fig. 7a). This 6.5 week stimulation-training delay ensures that additional neurons, produced as a consequence of stimulation, are sufficiently mature ( $\sim 6$ weeks old) to contribute to spatial learning [previous experiment (Kee et al., 2007b; Stone et al., 2010)]. During training, latency to find the platform declined similarly in both groups (Fig. 7b; Day $\times$ Stimulation ANOVA, significant main effect of Day only, $\left.F_{(2,49)}=51.23, p<0.01\right)$. Immediately after the completion of training, spatial memory was assessed in a probe test. In this test, both stimulated and nonstimulated mice searched selectively (Fig. 7c). However, mice in the stimulation group spent significantly more time in the target zone (Fig. $7 d$; unpaired $t$ test, $\left.t_{(49)}=2.02, p<0.05\right)$ and crossed the former platform location more frequently (Fig. 7e; unpaired $t$ test, $\left.t_{(49)}=3.83, p<0.01\right)$ compared with nonstimulated mice, indicating that stimulation of the EC facilitated spatial learning.

The beneficial effect of EC stimulation on spatial learning was detected in the probe test but not in the training latency data. Because the adoption of either localized/spatially precise (e.g., focal searching) or some nonlocalized/spatially imprecise (e.g., chaining) search strategies may contribute to reduced escape latencies across training (Gallagher et al., 1993; Lipp and Wolfer, 1998; Wolfer et al., 1998; Clapcote and Roder, 2004), latency data can be poor predictors of spatial learning (Clapcote and Roder, 2004). To address whether stimulated and nonstimulated mice adopted different search strategies during training, we conducted a detailed analysis of search paths. Using tracking data and a criterion-based algorithm (Garthe et al., 2009), search paths were objectively classified into eight mutually exclusive categories (Fig. $7 f$ ). At the beginning of training, thigmotaxis and random search strategies predominated in both groups, accounting for $>75 \%$ of paths (Fig. $7 g$ ). In contrast, by the end of training, these spatially imprecise strategies were progressively replaced by more localized/spatially precise strategies in both stimulated (Friedman's test, $\chi_{(8)}^{2}=$ 73.89, $p<0.01$ ) and control (Friedman's test, $\left.\chi_{(8)}^{2}=66.66, p<0.01\right)$ groups. Most strikingly, the overall frequencies of search strategies differed between groups ( $\chi^{2}$ test of independence, $\chi_{(7)}^{2}=6.39, p<$ 0.05). This difference was most apparent by an elevated prevalence of direct swims and focal searches in stimulated relative to nonstimulated mice (Fig. $7 h$ ). Therefore, consistent with the probe test, these analyses provide convergent evidence that stimulation of the EC facilitated spatial learning by promoting the adoption of localized/spatially precise search strategies.

Whether this stimulation-induced facilitation of spatial learning is mediated by a stimulation-induced enhancement of adult neurogenesis or some other mechanism is not clear. Consistent with previous studies (Kee et al., 2007b; Stone et al., 2010), we showed that 6-week-old, but not 1-week-old, adult-generated neurons are incorporated into DG circuits engaged during spatial learning (Fig. 6). Therefore, the beneficial effects of EC stimula- 
tion would be expected to have a delayed onset if they are mediated by a neurogenic mechanism. To test this, we next trained mice 1.5 weeks, rather than 6.5 weeks, after stimulation of the EC (Fig. 8a). As before, both stimulated and control mice required progressively less time to find the platform during training, and there were no differences between groups (Fig. $8 b$; Day $\times$ Stimulation ANOVA, significant main effect of Day only, $F_{(2,49)}=64.14$, $p<0.01)$. Furthermore, both stimulated and control mice progressed from predominantly nonlocalized/spatially imprecise strategies (e.g., thigmotaxis and random search) at the start of training to predominantly localized/spatially precise strategies (e.g., direct swim, focal search, or directed search) at the completion of training (Fig. $8 c$; Friedman's test for Stimulated Group, $\chi_{(8)}^{2}=76.34, p<0.01$; Friedman's test for Nonstimulated Group, $\left.\chi_{(8)}^{2}=94.72, p<0.01\right)$. In contrast to the mice trained 6.5 weeks after stimulation, our detailed analyses of swim paths during training revealed no differences in the prevalence of various search strategies between groups (Fig. $8 d ; \chi^{2}$ test of independence, $\left.\chi_{(7)}^{2}=3.30, p=0.86\right)$. Consistent with this, in the probe test $1 \mathrm{~d}$ after the completion of training, both stimulated and control mice searched selectively (Fig. $8 e$ ), and the degree of selectivity did not differ between groups: both stimulated and nonstimulated mice spent equivalent time searching the target zone (Fig. $8 f$; unpaired $t$ test, $\left.t_{(49)}=1.11, p=0.27\right)$ and crossed the former platform location with similar frequency (Fig. $8 g$; unpaired $t$ test, $\left.t_{(49)}=0.60, p=0.55\right)$. Therefore, spatial learning is not facilitated when training takes place 1.5 weeks after stimulation, suggesting that the beneficial effects of stimulation are delayed in their onset.

These data strengthen the causal link between stimulation-induced increases in adult neurogenesis and facilitated spatial learning. Six-week-old (but not 1-weekold) adult-generated neurons are engaged during spatial learning. Stimulation increases neurogenesis, and the facilitatory effects of stimulation-induced changes in neurogenesis do not emerge until 6.5 weeks after stimulation. As an additional test of this idea, an additional group of mice received stimulation after (rather than before) training in the water maze (Fig. 8h). It is unlikely that new neurons would be retroactively integrated into dentate circuits supporting spatial memory. Therefore, it is unlikely that new neurons, generated as a consequence of stimulation, would contribute to spatial learning in this experiment. As expected, during training, both groups of mice required progressively less time to locate the

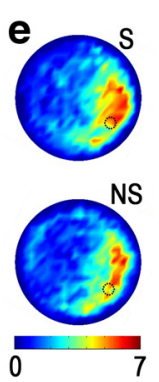

h

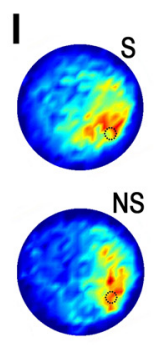

b
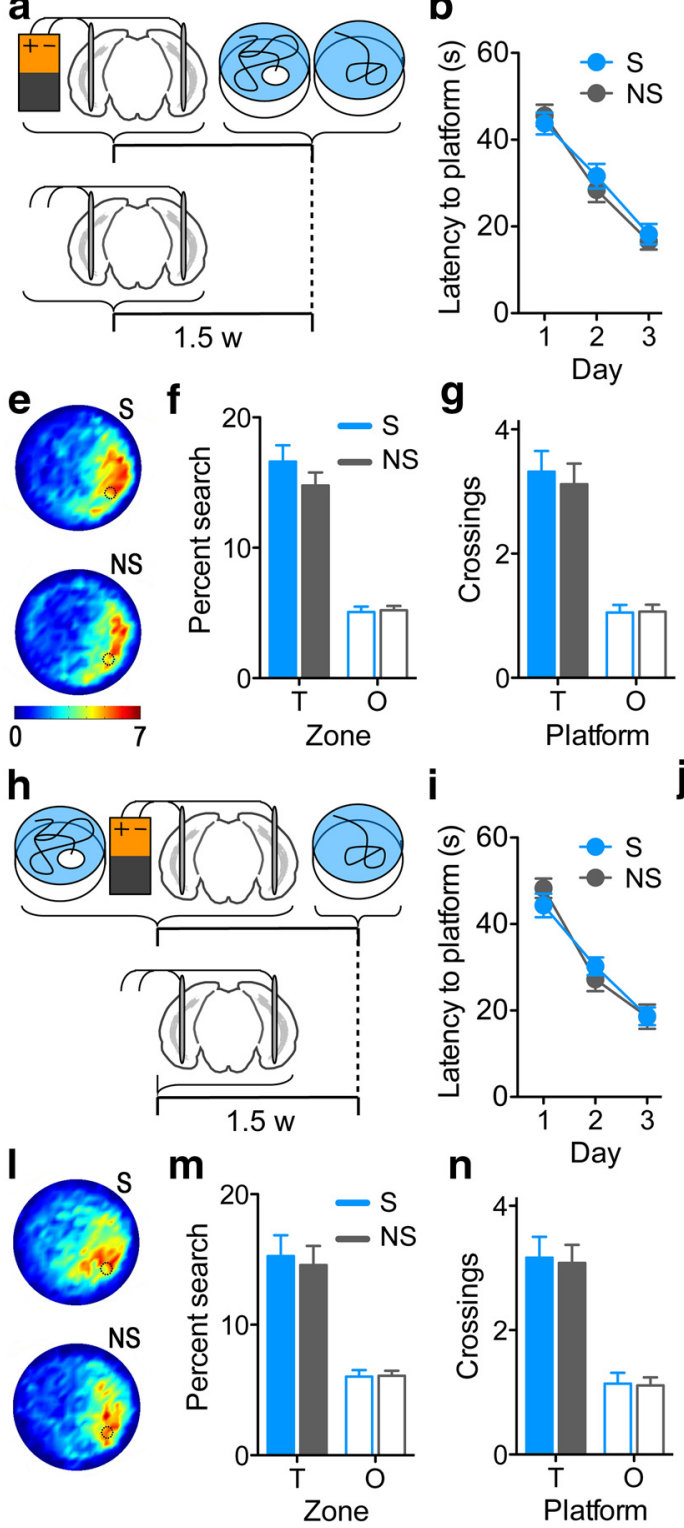

C
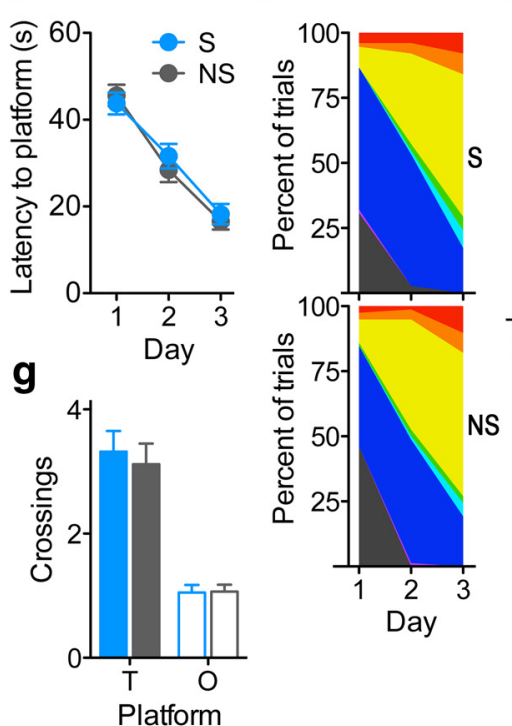

d
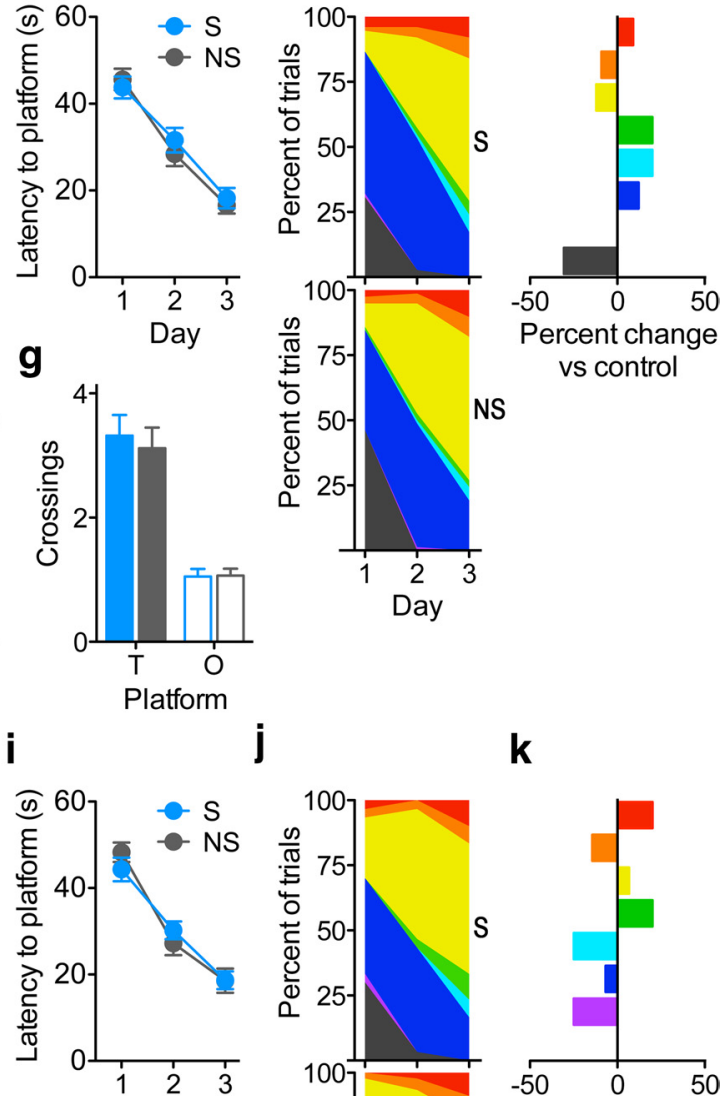

j

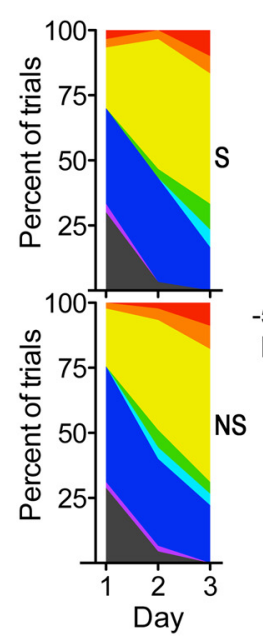

$\mathbf{k}$

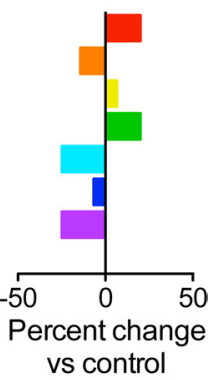

Figure 8. Specificity of stimulation-induced enhancement of spatial memory. $\boldsymbol{a}$, At 1.5 weeks after bilateral stimulation $(n=$ 25) or electrode insertion only $(n=26)$, mice were trained in the water maze and spatial memory was assessed $1 \mathrm{~h}$ after the completion of training. $\boldsymbol{b}$, Latencies to reach the platform declined equivalently for stimulated (S) and nonstimulated (NS) mice during the training period. c, Percentage of trials classified in each search strategy category across training days for stimulated (left graph) and nonstimulated (right graph) mice. $\boldsymbol{d}$, Percentage difference in the frequency of each search strategy in stimulated relative to nonstimulated mice, indicating minimal variability between groups. $\boldsymbol{e}$, Mice searched selectively in the probe test. Density plots for grouped data, with accompanying color scale, represent the number of visits per mouse per $5 \times 5 \mathrm{~cm}$ area. $\boldsymbol{f}, \boldsymbol{g}$, Percentage time searching the target zone (T) compared with other $(0)$ zones $(\boldsymbol{f})$ and target platform crossings (T) compared with other platform locations $(0)(\boldsymbol{g})$ for stimulated (S) versus nonstimulated (NS) mice in the probe test. $\boldsymbol{h}$, Separate groups of mice received bilateral stimulation $(n=24)$ or electrode insertion only $(n=24) 1 \mathrm{~d}$ after the completion of water-maze training, and spatial memory was assessed 1.5 weeks after surgery. $\boldsymbol{i}$, Latencies to reach the platform declined equivalently for both groups during the training period. $j$, Percentage of trials classified in each search strategy category across training days for stimulated (left graph) and nonstimulated (right graph) mice. $\boldsymbol{k}$, Percentage difference in the frequency of each search strategy in stimulated relative to nonstimulated mice, indicating minimal variability between groups. $I$, Mice searched selectively in the probe test. Density plots for grouped data, with accompanying color scale, represent the number of visits per mouse per $5 \times 5 \mathrm{~cm}$ area. $\boldsymbol{m}, \boldsymbol{n}$, Both groups spent more time searching the target zone $(\boldsymbol{m})$ and crossing the target platform location $(\boldsymbol{n})$ compared with other zones and platform locations, respectively; however, there were no differences in these measures between groups.

platform (Fig. 8i; Day $\times$ Stimulation ANOVA, significant main effect of Day only, $\left.F_{(2,46)}=69.96, p<0.01\right)$ and progressed from predominantly nonlocalized/spatially imprecise to localized/spatially precise strategies (Fig. 8j; Friedman's test for Stimulated Group, $\chi_{(8)}^{2}=40.43, p<0.01$; Friedman's test for Nonstimulated 

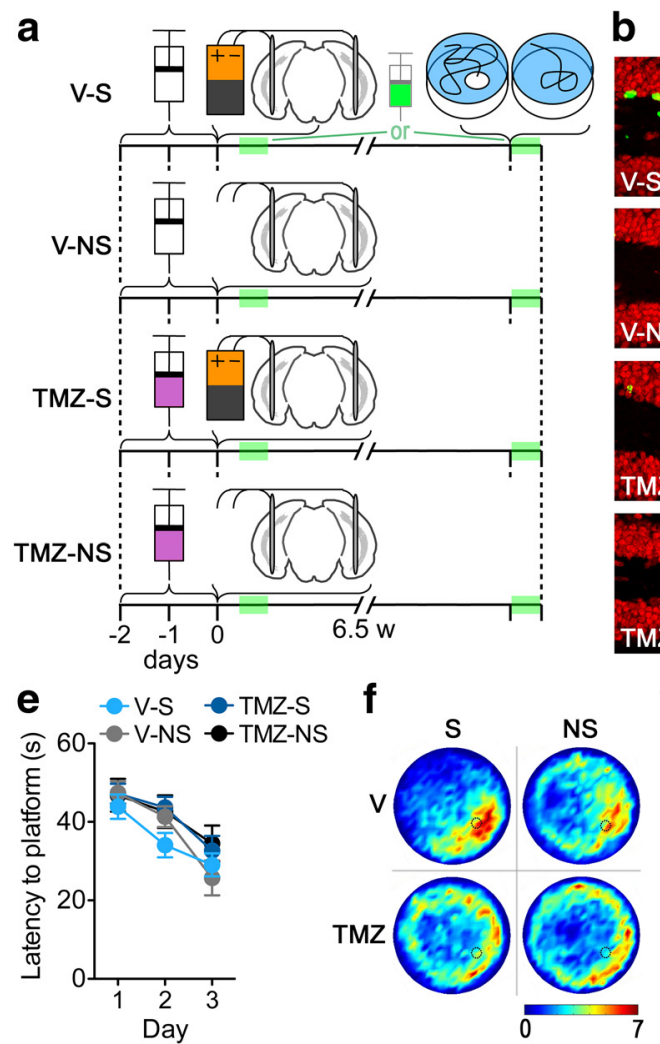

C

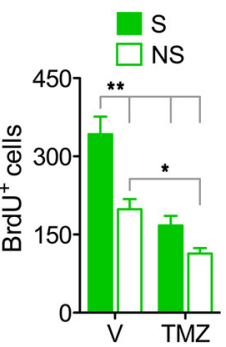

d

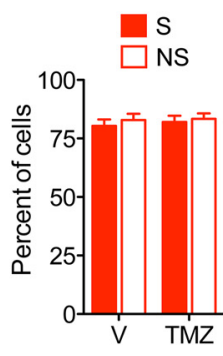

h

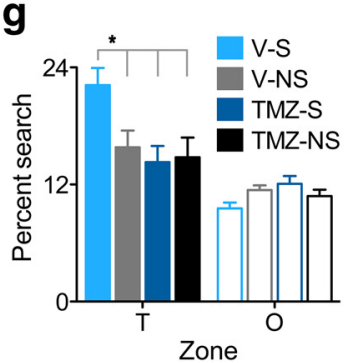

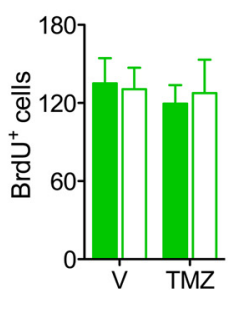

i

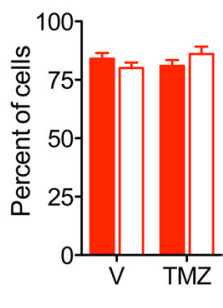

Figure 9. Suppressing the stimulation-induced increase in neurogenesis blocks spatial memory enhancement. $\boldsymbol{a}$, At $1 \mathrm{~d}$ after the completion of V or TMZ injections, four groups of mice underwent bilateral stimulation (S) or electrode insertion only (NS) (V-S, $n=24 ; \mathrm{V}-\mathrm{NS}, n=17 ; \mathrm{TMZ}-\mathrm{S}, n=24 ; \mathrm{TMZ}-\mathrm{NS}, n=17)$. Six and a half weeks after surgery, all mice were trained in the water maze, and spatial memory was assessed $1 \mathrm{~h}$ after the completion of training. Subsets of these mice received BrdU injections either during an early poststimulation period (V-S, $n=8 ; \mathrm{V}-\mathrm{NS}, n=8 ; \mathrm{TMZ}$-S, $n=5 ; \mathrm{TMZ}-\mathrm{NS}, n=5)$ or after the completion of probe testing (V-S, $n=5 ; \mathrm{V}-\mathrm{NS}, n=7 ; \mathrm{TMZ}-\mathrm{S}, n=7 ; \mathrm{TMZ}-\mathrm{NS}, n=5) \cdot \boldsymbol{b}, \mathrm{BrdU}^{+}$cells (green) labeled during the early poststimulation period (NeuN counterstain; scale bar, $50 \mu \mathrm{m}$ ). c, BrdU incorporation after labeling during the early poststimulation period. The stimulation-induced increase in proliferation was blocked by TMZ treatment. $\boldsymbol{d}$, Similar proportions of BrdU ${ }^{+}$cells were NeuN ${ }^{+}$in each group when mice received BrdU injections during the early poststimulation period.e, Latencies to reach the platform declined equivalently for all groups of mice during the training period. $\boldsymbol{f}$, Density plots for grouped data, with accompanying color scale, represent the number of visits per mouse per $5 \times 5 \mathrm{~cm}$ area. $\boldsymbol{f}, \boldsymbol{g}, \mathrm{V}$-S mice spent significantly more time searching the target (T) zone compared with all other groups. $\boldsymbol{h}$, BrdU incorporation after behavioral tests was similar across groups. $\boldsymbol{i}$, Similar proportions of these BrdU ${ }^{+}$cells costained for NeuN across groups. ${ }^{*} p<0.05,{ }^{* *} p<0.01$.

Group, $\chi_{(8)}^{2}=46.02, p<0.01$ ) with equivalent tendency (Fig. $8 k$; $\chi^{2}$ test of independence, $\left.\chi_{(7)}^{2}=1.57, p=0.98\right)$. Similarly, in the subsequent probe test, both groups searched selectively in the region of the pool that formerly contained the platform (Fig. 8l). Because the degree of selectivity did not differ between groups (Fig. 8m, $n$; unpaired $t$ test for Zones, $t_{(46)}=0.32, p=0.75$; unpaired $t$ test for Platform Crossings, $t_{(46)}=0.19, p=0.85$ ), these results exclude the possibility that stimulation of the EC nonspecifically facilitates memory retrieval. Moreover, they also indicate that stimulation does not disrupt the expression of a previously acquired memory.

\section{Facilitation of spatial memory is prevented by blocking adult neurogenesis}

To more directly evaluate whether EC stimulation facilitates the formation of spatial memory via a neurogenic mechanism, we next examined the impact of inhibiting neurogenesis using the DNA-alkylating agent TMZ (Garthe et al., 2009). In this experiment, stimulated or nonstimulated control mice were pretreated with V or TMZ. Six and a half weeks later, they were trained in the water maze (three trials per $\mathrm{d}$ for $3 \mathrm{~d}$ ), and then spatial memory was assessed in a probe test $1 \mathrm{~h}$ after the completion of training (Fig. 9a). To assess neurogenesis at different poststimulation time points, mice were additionally treated with BrdU either 3-5 d postoperatively (i.e., a time point corresponding to the peak in stimulation-induced changes in proliferation) or after watermaze testing (a time point when proliferation rates would be expected to have returned to baseline or $\sim 7$ weeks postoperatively). In mice treated with BrdU soon after training, BrdU incorporation differed between groups (Fig. 9b,c; ANOVA Group effect, $\left.F_{(3,22)}=14.91, p<0.001\right)$, indicating that proliferation was altered at this early poststimulation time point. Most notably, in V-treated mice, EC stimulation increased proliferation, as we had observed previously (Duncan's post hoc test, $p<0.01$ ), and this stimulation-induced increase was attenuated by TMZ treatment (TMZ-S $<$ V-S, Duncan's post hoc test, $p<0.01$ ). Additionally, TMZ treatment reduced proliferation by $\sim 45 \%$ in nonstimulated mice (Duncan's post hoc test, $p<0.05$ ), as expected. There were no group differences in the proportion of BrdU-labeled cells expressing the neuronal marker NeuN (Fig. $9 d$; ANOVA Group effect, $F_{(3,22)}=0.25, p=0.86$ ), indicating that these treatment combinations did not alter the fate of newly generated cells.

During training, latency to find the platform declined similarly in all groups (Fig. $9 e$; Day $\times$ Group ANOVA, significant main effect of Day only, $\left.F_{(2,156)}=27.54, p<0.001\right)$. However, in the probe test after training, the $\mathrm{V}$-treated, stimulated group outperformed all other groups, spending more time in the target zone (Fig. $9 f, g$; ANOVA on target zone data, Group effect, $F_{(3,78)}=$ $4.69, p<0.01$, Duncan's post hoc tests, $p<0.05$ ). These data there- 
fore replicate our previous finding that EC stimulation facilitates spatial memory formation (V-S group $>\mathrm{V}-\mathrm{NS}$ group) and, most importantly, show that this enhancement is prevented when the stimulation-induced enhancement of neurogenesis is blocked. Both TMZ and stimulation only transiently altered neurogenesis levels because BrdU incorporation was equivalent across groups in mice treated with BrdU after behavioral testing (Fig. $9 h$; ANOVA Group effect, $\left.F_{(3,20)}=0.13, p=0.94\right)$, and there were no group differences in the proportion of BrdU-labeled cells expressing the neuronal marker NeuN (Fig. 9i; ANOVA Group effect, $\left.F_{(3,20)}=1.06, p=0.39\right)$.

\section{Discussion}

Although DBS targeting hippocampal afferents may slow or arrest cognitive decline (Laxton et al., 2010), the underlying mechanisms of action are poorly understood. Using a mouse model, we provide four lines of evidence that such pro-cognitive effects are mediated, at least in part, by activity-dependent promotion of hippocampal neurogenesis. First, using parameters analogous to clinical high-frequency DBS, we show that targeted stimulation of EC in adult mice produces an anatomically and temporally specific increase in DGC production. Relative to cells produced under basal conditions, stimulation-induced cells exhibit comparable rates of neuronal differentiation and long-term survival. Second, new neurons produced as a consequence of EC stimulation assume functional roles in hippocampal circuits. Relative to cells produced under basal conditions, they show comparable localization in the DG, mature normally, and integrate into hippocampal networks engaged by spatial memory in a maturationdependent manner. Third, acute, bilateral stimulation of the EC facilitated spatial memory formation. Facilitation only emerged several weeks after stimulation and not if stimulation occurred $\sim 1$ week before or after training. This time course tracks exactly the maturation-dependent time course of integration of adultgenerated neurons into spatial memory circuits. Fourth, facilitation of spatial memory formation was prevented by blocking neurogenesis, suggesting that the pro-cognitive effects of EC stimulation are most likely mediated by a neurogenic mechanism.

We found that stimulation for $1 \mathrm{~h}$ was sufficient to promote proliferation in the DG. The pro-proliferative effects of EC stimulation had distinct temporal and anatomical profiles. Similar to electroconvulsive shock (Ma et al., 2009), induced seizures (Parent et al., 1997; Jiang et al., 2003), and targeted brain stimulation (Bruel-Jungerman et al., 2006; Toda et al., 2008), increased proliferation occurred in a delayed manner and peaked 3-5 d after stimulation before returning to baseline. Furthermore, after unilateral stimulation, increased proliferation was limited to the side ipsilateral to the electrode site. This is consistent with previous studies showing that the perforant path projection from layer II medial/lateral EC neurons is almost exclusively ipsilateral in the mouse (van Groen et al., 2002, 2003; Amaral and Lavenex, 2007).

Similar to previous studies, we found that stimulation did not alter rates of neuronal differentiation or apoptosis, suggesting that these effects are most likely mediated instead by increasing the proliferative activity of neural progenitor cells in the subgranular zone (Bruel-Jungerman et al., 2006; Toda et al., 2008; Kitamura et al., 2010; Encinas et al., 2011). The subgranular zone contains cells with varying degrees of multipotency and capacities for self-renewal and proliferation. The most proliferative of these are type 2 progenitor cells, whose progeny progressively differentiate into DGCs. Stimulation of another limbic target, the anterior thalamic nucleus, promotes adult neurogenesis in the subgranular zone by increasing the number and mitotic activity of type 2 progenitor cells (Encinas et al., 2011), leading to increased DGC production (Toda et al., 2008; Encinas et al., 2011). Similarly, other pro-neurogenic stimuli, such as seizures and electroconvulsive shock, primarily increase neurogenesis through increased progenitor cell proliferation, suggesting shared underlying mechanisms (Parent and Murphy, 2008; Zhao et al., 2008).

Under basal conditions, granule cells generated during adulthood take several weeks to fully mature and establish functional afferent and efferent connections (Zhao et al., 2006; Toni et al., 2007, 2008). Using a retroviral strategy to label adult-generated granule cells, our data indicate that neuronal maturation follows an equivalent time course after unilateral EC stimulation: one week after infection, retrovirally labeled cells typically had short, aspiny, dendritic processes and axons that had not yet reached the CA3 region, whereas 6 weeks after infection, retrovirally labeled cells had mature neuronal morphology, with spiny, highly arborized dendritic processes extending into the molecular layer and axons extending into CA3. Because labeled neurons had similar properties both ipsilateral and contralateral to the stimulation site, these data suggest that neurons produced as a consequence of stimulation acquire normal morphology and likely establish normal afferent and efferent connections. Other conditions, such as epileptic activity (Parent et al., 1997; Jessberger et al., 2007), may also lead to a sustained increase in adult hippocampal neurogenesis. However, after epileptic activity, newly generated neurons develop abnormal morphology, and many become ectopically located in the hilus. In contrast, we found no evidence for abnormal morphology or ectopic localization after acute stimulation, suggesting that the low-current intensity stimulation that we used does not promote the aberrant integration of adult-generated neurons into hippocampal circuits.

Previous studies have shown that, once sufficiently mature, adult-generated neurons are activated during memory formation and/or expression (Kee et al., 2007b; Tashiro et al., 2007; Trouche et al., 2009; Stone et al., 2010). In our experiments, EC stimulation nearly doubled levels of neurogenesis, and there was a corresponding increase in the numbers of adult-generated neurons in the pool of granule cells activated by the expression of a watermaze memory. This suggests that stimulation-induced neurons become functionally integrated into hippocampal networks supporting spatial memory. Importantly, this increase was specific to neurons generated 3-5 d after stimulation and limited to the side ipsilateral to the stimulation site and only when stimulation preceded training by 6.5 weeks. This finding is consistent with previous studies showing that integration of newborn DGCs into hippocampal circuits supporting water-maze memory is regulated by cell age, with newborn DGCs not maximally contributing until they are 5 weeks or older (Kee et al., 2007b; Stone et al., 2010). This delayed time course for functional integration follows the establishment and gradual maturation of excitatory connections of newborn DGCs (Piatti et al., 2006; Zhao et al., 2008).

Most strikingly, bilateral stimulation of the EC facilitated the formation of a hippocampus-dependent water-maze memory. In the probe test after training, stimulated mice searched more selectively compared with nonstimulated controls. Detailed analyses of swim paths during training revealed that stimulated mice were more likely to use localized/spatially precise search strategies (e.g., direct swim, focal search) and that the increased frequency of these more effective strategies very likely accounts for the improved spatial memory formation. In these types of experiments, a key question is whether improved memory formation is causally related to changes in neurogenesis. For example, although environmental enrichment both promotes adult neuro- 
genesis and facilitates memory formation, its memory effects are independent of those on neurogenesis (Meshi et al., 2006). In our experiments, stimulation of the EC may produce a range of other effects that, in principle, could contribute to improved spatial memory formation. These might include effects on developmentally generated hippocampal neurons (e.g., dendritic remodeling) and/or on the differentiation and maturation of existing adult-generated granule cells. However, memory facilitation was fully blocked by pharmacological blockade of the stimulationinduced increase in neurogenesis. Because TMZ would predominantly affect proliferation (and not postmitotic cells), this experiment reduces the likelihood that these non-neurogenic mechanisms contribute to the pro-cognitive effects of EC stimulation. One related concern is that TMZ treatment may impact general health. However, TMZ treatment did not impair spatial memory formation in nonstimulated mice. Moreover, even more prolonged TMZ treatment does not affect weight, induce neuroinflammation, or alter general behavioral or hematological profiles in mice (Garthe et al., 2009), and so it seems unlikely that these potential off-target effects confound our results.

Our experiments suggest that promotion of adult neurogenesis is one plausible mechanism by which DBS might exert procognitive effects. In translating these findings to clinical settings, several issues are worth considering. First, mechanisms underlying the pro-cognitive effects of DBS likely include, but need not be limited to, activity-dependent promotion of adult neurogenesis. For example, stimulation may induce neurotransmitter release and local or transsynaptic modulation of neural activity at the cellular level (Kringelbach et al., 2007), as well as restore basal activity levels within dysregulated brain regions at the circuit level (Mayberg et al., 2005; Laxton et al., 2010). Such effects might act in concert with stimulation-induced changes in neurogenesis to promote cognitive recovery. DBS of hippocampal afferents may additionally have immediate effects on memory [e.g., vivid autobiographical recall seen during initiation of stimulation in some of our patients (Hamani et al., 2008; Laxton et al., 2010)], and these are unlikely to be mediated by a neurogenic mechanism. Second, pro-neurogenic effects of stimulation are not limited to the EC. For example, stimulation of other limbic targets increases adult neurogenesis (Derrick et al., 2000; Toda et al., 2008; Encinas et al., 2011). Whether such stimulation has beneficial effects on cognitive function remains to be determined. Indeed, stimulation of the anterior thalamic nucleus may impair, rather than facilitate, cognitive function in rats (Hamani et al., 2010), and these effects are consistent with memory impairments reported in some DBS-implanted epilepsy patients (Fisher et al., 2010). Third, although we used short-duration stimulation in our experiments, in clinical settings DBS typically involves chronic intermittent or continuous stimulation. The main advantage of using acute stimulation was that it allowed us to isolate effects of stimulation-induced neurogenesis on behavior. Although more sustained stimulation might bring greater cognitive benefit, it is important to recognize that chronic EC stimulation, albeit likely at higher intensity than used here, carries a greater risk of toxicity (Sloviter et al., 1996).

\section{References}

Amaral D, Lavenex P (2007) Hippocampal neuroanatomy. In: The hippocampus book, Ed 1 (Anderson P, Morris RG, Amaral DG, Bliss T, O'Keefe J, eds). New York: Oxford UP.

Bath KG, Mandairon N, Jing D, Rajagopal R, Kapoor R, Chen ZY, Khan T, Proenca CC, Kraemer R, Cleland TA, Hempstead BL, Chao MV, Lee FS (2008) Variant brain-derived neurotrophic factor (Val66Met) alters adult olfactory bulb neurogenesis and spontaneous olfactory discrimination. J Neurosci 28:2383-2393.

Brandt MD, Jessberger S, Steiner B, Kronenberg G, Reuter K, Bick-Sander A, von der Behrens W, Kempermann G (2003) Transient calretinin expression defines early postmitotic step of neuronal differentiation in adult hippocampal neurogenesis of mice. Mol Cell Neurosci 24:603-613.

Bruel-Jungerman E, Davis S, Rampon C, Laroche S (2006) Long-term potentiation enhances neurogenesis in the adult dentate gyrus. J Neurosci 26:5888-5893.

Chun SK, Sun W, Park JJ, Jung MW (2006) Enhanced proliferation of progenitor cells following long-term potentiation induction in the rat dentate gyrus. Neurobiol Learn Mem 86:322-329.

Clapcote SJ, Roder JC (2004) Survey of embryonic stem cell line source strains in the water maze reveals superior reversal learning of 129S6/ SvEvTac mice. Behav Brain Res 152:35-48.

Davis KD, Taub E, Houle S, Lang AE, Dostrovsky JO, Tasker RR, Lozano AM (1997) Globus pallidus stimulation activates the cortical motor system during alleviation of parkinsonian symptoms. Nat Med 3:671-674.

Deng W, Aimone JB, Gage FH (2010) New neurons and new memories: how does adult hippocampal neurogenesis affect learning and memory? Nat Rev Neurosci 11:339-350.

Derrick BE, York AD, Martinez JL Jr (2000) Increased granule cell neurogenesis in the adult dentate gyrus following mossy fiber stimulation sufficient to induce long-term potentiation. Brain Res 857:300-307.

Deuschl G, Schade-Brittinger C, Krack P, Volkmann J, Schäfer H, Bötzel K, Daniels C, Deutschländer A, Dillmann U, Eisner W, Gruber D, Hamel W, Herzog J, Hilker R, Klebe S, Kloss M, Koy J, Krause M, Kupsch A, Lorenz D, et al. (2006) A randomized trial of deep-brain stimulation for Parkinson's disease. N Engl J Med 355:896-908.

Encinas JM, Hamani C, Lozano AM, Enikolopov G (2011) Neurogenic hippocampal targets of deep brain stimulation. J Comp Neurol 519:6-20.

Fisher R, Salanova V, Witt T, Worth R, Henry T, Gross R, Oommen K, Osorio I, Nazzaro J, Labar D, Kaplitt M, Sperling M, Sandok E, Neal J, Handforth A, Stern J, DeSalles A, Chung S, Shetter A, Bergen D, et al. (2010) Electrical stimulation of the anterior nucleus of thalamus for treatment of refractory epilepsy. Epilepsia 51:899-908.

Gallagher M, Burwell R, Burchinal M (1993) Severity of spatial learning impairment in aging: development of a learning index for performance in the Morris water maze. Behav Neurosci 107:618-626.

Garthe A, Behr J, Kempermann G (2009) Adult-generated hippocampal neurons allow the flexible use of spatially precise learning strategies. PLoS One 4:e5464.

Gimsa J, Habel B, Schreiber U, van Rienen U, Strauss U, Gimsa U (2005) Choosing electrodes for deep brain stimulation experiments-electrochemical considerations. J Neurosci Methods 142:251-265.

Gimsa U, Schreiber U, Habel B, Flehr J, van Rienen U, Gimsa J (2006) Matching geometry and stimulation parameters of electrodes for deep brain stimulation experiments: numerical considerations. J Neurosci Methods 150:212-227.

Gould E, Tanapat P (1997) Lesion-induced proliferation of neuronal progenitors in the dentate gyrus of the adult rat. Neuroscience 80:427-436.

Hamani C, McAndrews MP, Cohn M, Oh M, Zumsteg D, Shapiro CM, Wennberg RA, Lozano AM (2008) Memory enhancement induced by hypothalamic/fornix deep brain stimulation. Ann Neurol 63:119-123.

Hamani C, Dubiela FP, Soares JC, Shin D, Bittencourt S, Covolan L, Carlen PL, Laxton AW, Hodaie M, Stone SS, Ha Y, Hutchison WD, Lozano AM, Mello LE, Oliveira MG (2010) Anterior thalamus deep brain stimulation at high current impairs memory in rats. Exp Neurol 225:154-162.

Jessberger S, Zhao C, Toni N, Clemenson GD Jr, Li Y, Gage FH (2007) Seizure-associated, aberrant neurogenesis in adult rats characterized with retrovirus-mediated cell labeling. J Neurosci 27:9400-9407.

Jiang W, Wan Q, Zhang ZJ, Wang WD, Huang YG, Rao ZR, Zhang X (2003) Dentate granule cell neurogenesis after seizures induced by pentylenetrazol in rats. Brain Res 977:141-148.

Kee N, Teixeira CM, Wang AH, Frankland PW (2007a) Imaging activation of adult-generated granule cells in spatial memory. Nat Protoc 2:3033-3044.

Kee N, Teixeira CM, Wang AH, Frankland PW (2007b) Preferential incorporation of adult-generated granule cells into spatial memory networks in the dentate gyrus. Nat Neurosci 10:355-362.

Kitamura T, Saitoh Y, Murayama A, Sugiyama H, Inokuchi K (2010) LTP induction within a narrow critical period of immature stages enhances the 
survival of newly generated neurons in the adult rat dentate gyrus. Mol Brain 3:13.

Koller W, Pahwa R, Busenbark K, Hubble J, Wilkinson S, Lang A, Tuite P, Sime E, Lazano A, Hauser R, Malapira T, Smith D, Tarsy D, Miyawaki E, Norregaard T, Kormos T, Olanow CW (1997) High-frequency unilateral thalamic stimulation in the treatment of essential and parkinsonian tremor. Ann Neurol 42:292-299.

Kringelbach ML, Jenkinson N, Owen SL, Aziz TZ (2007) Translational principles of deep brain stimulation. Nat Rev Neurosci 8:623-635.

Laplagne DA, Espósito MS, Piatti VC, Morgenstern NA, Zhao C, van Praag H, Gage FH, Schinder AF (2006) Functional convergence of neurons generated in the developing and adult hippocampus. PLoS Biol 4:e409.

Laplagne DA, Kamienkowski JE, Espósito MS, Piatti VC, Zhao C, Gage FH, Schinder AF (2007) Similar GABAergic inputs in dentate granule cells born during embryonic and adult neurogenesis. Eur J Neurosci 25:2973-2981.

Laxton AW, Tang-Wai DF, McAndrews MP, Zumsteg D, Wennberg R, Keren R, Wherrett J, Naglie G, Hamani C, Smith GS, Lozano AM (2010) A phase I trial of deep brain stimulation of memory circuits in Alzheimer's disease. Ann Neurol 68:521-534.

Lipp HP, Wolfer DP (1998) Genetically modified mice and cognition. Curr Opin Neurobiol 8:272-280.

Ma DK, Jang MH, Guo JU, Kitabatake Y, Chang ML, Pow-Anpongkul N, Flavell RA, Lu B, Ming GL, Song H (2009) Neuronal activity-induced Gadd45b promotes epigenetic DNA demethylation and adult neurogenesis. Science 323:1074-1077.

Madsen TM, Treschow A, Bengzon J, Bolwig TG, Lindvall O, Tingström A (2000) Increased neurogenesis in a model of electroconvulsive therapy. Biol Psychiatry 47:1043-1049.

Madsen TM, Yeh DD, Valentine GW, Duman RS (2005) Electroconvulsive seizure treatment increases cell proliferation in rat frontal cortex. Neuropsychopharmacology 30:27-34.

Mallet L, Polosan M, Jaafari N, Baup N, Welter ML, Fontaine D, du Montcel ST, Yelnik J, Chéreau I, Arbus C, Raoul S, Aouizerate B, Damier P, Chabardès S, Czernecki V, Ardouin C, Krebs MO, Bardinet E, Chaynes P, Burbaud P, Cornu P, et al. (2008) Subthalamic nucleus stimulation in severe obsessive-compulsive disorder. N Engl J Med 359:2121-2134.

Mayberg HS, Lozano AM, Voon V, McNeely HE, Seminowicz D, Hamani C, Schwalb JM, Kennedy SH (2005) Deep brain stimulation for treatmentresistant depression. Neuron 45:651-660.

McCreery DB, Agnew WF, Yuen TG, Bullara L (1990) Charge density and charge per phase as cofactors in neural injury induced by electrical stimulation. IEEE Trans Biomed Eng 37:996-1001.

Meshi D, Drew MR, Saxe M, Ansorge MS, David D, Santarelli L, Malapani C, Moore H, Hen R (2006) Hippocampal neurogenesis is not required for behavioral effects of environmental enrichment. Nat Neurosci 9:729-731.

Ming GL, Song H (2005) Adult neurogenesis in the mammalian central nervous system. Annu Rev Neurosci 28:223-250.

Parent JM, Murphy GG (2008) Mechanisms and functional significance of aberrant seizure-induced hippocampal neurogenesis. Epilepsia 49 [Suppl 5]:19-25.

Parent JM, Yu TW, Leibowitz RT, Geschwind DH, Sloviter RS, Lowenstein DH (1997) Dentate granule cell neurogenesis is increased by seizures and contributes to aberrant network reorganization in the adult rat hippocampus. J Neurosci 17:3727-3738.

Parent JM, Valentin VV, Lowenstein DH (2002) Prolonged seizures increase proliferating neuroblasts in the adult rat subventricular zoneolfactory bulb pathway. J Neurosci 22:3174-3188.

Parent JM, Elliott RC, Pleasure SJ, Barbaro NM, Lowenstein DH (2006) Aberrant seizure-induced neurogenesis in experimental temporal lobe epilepsy. Ann Neurol 59:81-91.

Paxinos G, Franklin KBJ (2000) The mouse brain in stereotaxic coordinates, Ed 2. San Diego: Academic.

Piatti VC, Espósito MS, Schinder AF (2006) The timing of neuronal development in adult hippocampal neurogenesis. Neuroscientist 12:463-468.

Ponce FA, Lozano AM (2010) Deep brain stimulation state of the art and novel stimulation targets. Prog Brain Res 184:311-324.

Shahed J, Poysky J, Kenney C, Simpson R, Jankovic J (2007) GPi deep brain stimulation for Tourette syndrome improves tics and psychiatric comorbidities. Neurology 68:159-160.

Shors TJ (2008) From stem cells to grandmother cells: how neurogenesis relates to learning and memory. Cell Stem Cell 3:253-258.

Sloviter RS, Dean E, Sollas AL, Goodman JH (1996) Apoptosis and necrosis induced in different hippocampal neuron populations by repetitive perforant path stimulation in the rat. J Comp Neurol 366:516-533.

Stone SS, Teixeira CM, Zaslavsky K, Wheeler AL, Martinez-Canabal A, Wang AH, Sakaguchi M, Lozano AM, Frankland PW (2010) Functional convergence of developmentally and adult-generated granule cells in dentate gyrus circuits supporting hippocampus-dependent memory. Hippocampus. Advance online publication. Retrieved August 6, 2011. doi:10.1002/hipo.20845.

Tashiro A, Zhao C, Gage FH (2006) Retrovirus-mediated single-cell gene knockout technique in adult newborn neurons in vivo. Nat Protoc 1:3049-3055

Tashiro A, Makino H, Gage FH (2007) Experience-specific functional modification of the dentate gyrus through adult neurogenesis: a critical period during an immature stage. J Neurosci 27:3252-3259.

Teixeira CM, Pomedli SR, Maei HR, Kee N, Frankland PW (2006) Involvement of the anterior cingulate cortex in the expression of remote spatial memory. J Neurosci 26:7555-7564.

Toda H, Hamani C, Fawcett AP, Hutchison WD, Lozano AM (2008) The regulation of adult rodent hippocampal neurogenesis by deep brain stimulation. J Neurosurg 108:132-138.

Toni N, Teng EM, Bushong EA, Aimone JB, Zhao C, Consiglio A, van Praag H, Martone ME, Ellisman MH, Gage FH (2007) Synapse formation on neurons born in the adult hippocampus. Nat Neurosci 10:727-734.

Toni N, Laplagne DA, Zhao C, Lombardi G, Ribak CE, Gage FH, Schinder AF (2008) Neurons born in the adult dentate gyrus form functional synapses with target cells. Nat Neurosci 11:901-907.

Tronel S, Fabre A, Charrier V, Oliet SH, Gage FH, Abrous DN (2010) Spatial learning sculpts the dendritic arbor of adult-born hippocampal neurons. Proc Natl Acad Sci U S A 107:7963-7968.

Trouche S, Bontempi B, Roullet P, Rampon C (2009) Recruitment of adultgenerated neurons into functional hippocampal networks contributes to updating and strengthening of spatial memory. Proc Natl Acad Sci U S A 106:5919-5924.

van Groen T (2001) Entorhinal cortex of the mouse: cytoarchitectonical organization. Hippocampus 11:397-407.

van Groen T, Wyss JM (1990) The connections of presubiculum and parasubiculum in the rat. Brain Res 518:227-243.

van Groen T, Kadish I, Wyss JM (2002) Species differences in the projections from the entorhinal cortex to the hippocampus. Brain Res Bull 57:553-556.

van Groen T, Miettinen P, Kadish I (2003) The entorhinal cortex of the mouse: organization of the projection to the hippocampal formation. Hippocampus 13:133-149.

Vega CJ, Peterson DA (2005) Stem cell proliferative history in tissue revealed by temporal halogenated thymidine analog discrimination. Nat Methods 2:167-169.

Vidailhet M, Vercueil L, Houeto JL, Krystkowiak P, Benabid AL, Cornu P, Lagrange C, Tézenas du Montcel S, Dormont D, Grand S, Blond S, Detante O, Pillon B, Ardouin C, Agid Y, Destée A, Pollak P (2005) Bilateral deep-brain stimulation of the globus pallidus in primary generalized dystonia. N Engl J Med 352:459-467.

Volkmann J, Moro E, Pahwa R (2006) Basic algorithms for the programming of deep brain stimulation in Parkinson's disease. Mov Disord 21 [Suppl 14]:S284-289.

Wolfer DP, Mohajeri HM, Lipp HP, Schachner M (1998) Increased flexibility and selectivity in spatial learning of transgenic mice ectopically expressing the neural cell adhesion molecule L1 in astrocytes. Eur J Neurosci 10:708-717.

Zhao C, Teng EM, Summers RG Jr, Ming GL, Gage FH (2006) Distinct morphological stages of dentate granule neuron maturation in the adult mouse hippocampus. J Neurosci 26:3-11.

Zhao C, Deng W, Gage FH (2008) Mechanisms and functional implications of adult neurogenesis. Cell 132:645-660. 